Boletín de la Sociedad Geológica Mexicana

VOLUMEN 62, NÚM. 1, 2010, P. 1-23

\title{
Técnicas de caracterización mineral y su aplicación en exploración y explotación minera
}

\author{
Joan Carles Melgarejo ${ }^{1 *}$, Joaquín A. Proenza ${ }^{1}$, Salvador Galí ${ }^{1}$, Xavier Llovet ${ }^{2}$ \\ 1 Departament de Cristal·lografia, Mineralogia i Dipòsits Minerals, Facultat de Geologia, Universitat de \\ Barcelona, Martí i Franquès, s/n, 08028, Barcelona \\ 2Serveis Científico Tècnics, Universitat de Barcelona. Lluís Solé i Sabarís, 1-3, 08028 Barcelona \\ *joan.carles.melgarejo.draper@ub.edu
}

\section{Resumen}

En este trabajo se presenta una síntesis de las técnicas analíticas más utilizadas en la caracterización mineral, y su aplicación a la exploración y explotación minera. Las técnicas han sido clasificadas en 2 grupos. El primer grupo incluye a las técnicas de mayor uso ("técnicas convencionales"): (i) difracción de polvo de rayos X y difracción cuantitativa de rayos X, (ii) Microscopio electrónico de barrido con analizador de energías (SEM-EDS), (iii) catodoluminiscencia, y iv) microsonda electrónica (EMPA). El segundo grupo abarca un grupo de técnicas menos accesible, y mucho más caras, (“técnicas no convencionales"): (i) Particle Induced X-Ray Emission (Micro-PIXE), (ii) Secondary Ion Mass Spectrometry (SIMS, (iii) Laser-Ablation-Inductively Coupled Plasma-Mass Spectrometry (LA$I C P-M S)$. La mayor parte de la compilación esta dedicada a las técnicas convencionales (DRX, SEM-EDS y EMP), las cuales pueden ser de mayor impacto en el campo de la pequeña minería.

Palabras Claves: Caracterización mineral, técnicas analíticas, Difracción de rayos X, microscopio electrónico, microsonda electrónica.

\begin{abstract}
This paper is an overview of the main analytical techniques used in mineral characterization, as well as their application to mining exploration and exploitation. Two groups of techniques have been established. The first group includes more frequently used techniques ("conventional techniques"): (i) X-ray powder diffraction and quantitative XRD, (ii) scanning electron microscopy-energy dispersive X-ray spectroscopy (SEM-EDS), (iii) cathodoluminiscence, and iv) electron probe microanalysis (EPMA). In contrast, the second group comprises less commonly used and more expensive techniques ("unconventional techniques"): (i) Particle Induced X-Ray Emission (Micro-PIXE), (ii) Secondary Ion Mass Spectrometry (SIMS), (iii) Laser-Ablation-Inductively Coupled Plasma-Mass Spectrometry (LA-ICP-MS).

The paper covers mainly the fundamentals of the most commonly used analytical techniques in mineral characterization (DRX, SEM-EDS, EMPA), and information on applications of these tools in mining exploration, which can be of major interest in small mining operations.
\end{abstract}

Key words: Mineral characterization, analytical techniques, X-ray diffraction, Scanning electron microscopy, electron probe micro analysis 
Melgarejo et al.

\section{Introducción}

Con frecuencia, el estudio que se realiza para la valoración de un depósito mineral implica tan sólo el estudio genérico de las leyes de los elementos que se espera puedan ser interesantes en el tipo de depósito, obviando el estudio mineralógico detallado de las asociaciones minerales. No obstante, un estudio detallado de la mineralogía no tiene tan sólo connotaciones académicas como a veces se cree, sino que puede ayudar a incrementar el valor añadido de la explotación, e incluso por sí sólo puede ayudar a descartar o confirmar el interés del yacimiento. Teniendo en cuenta el bajo costo de los análisis mineralógicos, cuando se comparan con otros métodos, no deja de ser sorprendente este comportamiento erróneo de muchas empresas.

El objetivo de este trabajo es el de ofrecer una revisión de las técnicas de caracterización mineral más usuales, así como valorar la incidencia de los estudios mineralógicos en la exploración, valoración y explotación de yacimientos minerales. Las técnicas han sido clasificadas en 2 grupos. El primer grupo incluye a las técnicas de mayor uso, de un coste económico relativamente bajo, las cuales podemos denominar "técnicas convencionales":

(i) Difracción de polvo de rayos X y difracción cuantitativa de rayos $\mathrm{X}$

(ii) Microscopio electrónico de barrido con analizador de energías (SEM-EDS)

(iii) Catodoluminiscencia

(iv) Microsonda electrónica (EMP)

El segundo grupo abarca un grupo de técnicas menos accesibles, y mucho más caras, las cuales denominamos en este trabajo "técnicas no convencionales":

(i) Particle Induced X-Ray Emission (Micro-PIXE)

(ii) Secondary Ion Mass Spectrometry (SIMS)

(iii) Laser-Ablation-Inductively Coupled PlasmaMass Spectrometry (LA-ICP-MS)

La mayor parte de esta compilación está dedicada a las técnicas convencionales (DRX, SEM-EDS y EMP), las cuales pueden ser de mayor impacto en el campo de la pequeña minería.

\section{Difracción de polvo cristalino}

La difracción de polvo es un método de análisis estructural, que permite identificar los minerales por su estructura cristalina. No se trata pues de un método analítico químico, pero, como veremos, permite indirectamente estimar la composición de la muestra analizada con una buena aproximación del contenido en elementos mayoritarios. Existe mucha documentación sobre el método; entre las compilaciones clásicas merecen citarse las de Klug y Alexander (1954), Bish y Post (1989) y Cullity y Stock (2001).

Prácticamente todos los minerales son cristalinos, es decir, disponen sus componentes químicos (átomos, iones, moléculas) de forma regular, llenando el espacio. Even- tualmente, todos los cristales pueden desarrollar formas exteriores poliédricas, aunque gran parte de la masa mineral no manifiesta esta propiedad necesariamente. La distribución regular en el espacio de los componentes del mineral se describe mediante las redes cristalinas, que ponen de manifiesto la repetición periódica de la celda del mineral. La celda es pues una unidad, en forma de paralelepípedo, que repetida idénticamente llena todo el espacio del cristal. La descripción de la celda proporciona toda la información sobre la estructura cristalina del mineral. Existen dos niveles de descripción de la celda: a) los parámetros del paralelepípedo, tres aristas y tres ángulos que denominamos a, b, $\mathrm{c}, \alpha, \beta, \gamma ; \mathrm{b})$ la disposición de los átomos en la celda. Cada mineral se caracteriza, en primer lugar, por sus parámetros de celda. Puede ocurrir que dos minerales distintos tengan parámetros de celda casi idénticos. En estos casos, para distinguirlos, hay que tener en cuenta el segundo nivel, es decir, qué átomos (composición) hay en la celda y cómo se disponen (estructura). Como veremos, la difracción de polvo cristalino nos informa sobre estos dos niveles y, por tanto, nos permite distinguir perfectamente minerales con redes similares. A continuación damos algunas nociones sobre el fenómeno de la difracción.

Las redes periódicas cristalinas están formadas por planos reticulares regularmente espaciados, de la misma forma que una red de dos dimensiones está formada por hileras de puntos regularmente espaciadas. Una red cristalina contiene planos reticulares en orientaciones diversas, que se notan mediante tres enteros ' $\mathrm{h}, \mathrm{k}, \mathrm{l}$ ”. El espaciado que corresponde a cada sucesión de planos paralelos hkl se indica mediante el símbolo $\mathrm{d}_{\mathrm{hk}}$. La ley de Bragg de la difracción permite obtener para cada mineral los valores de sus espaciados (por ejemplo $\mathrm{d}_{100}, \mathrm{~d}_{200}, \mathrm{~d}_{001}, \ldots . . \mathrm{d}_{573 \ldots}$ etc) $\mathrm{y}$ de este modo permitir su identificación. La ley de Bragg establece que los rayos $\mathrm{X}$ se reflejan sobre los planos reticulares de los cristales según un ángulo (ángulo $\theta$ ) que depende del espaciado $\mathrm{d}_{\mathrm{hkl}}$ : Ley de Bragg: $\lambda=2 \mathrm{~d}_{\mathrm{hkl}} \sin \theta$

Donde $\lambda$ es la longitud de onda de los rayos $\mathrm{X}$.

Cada mineral está caracterizado por una serie de valores de $\mathrm{d}_{\mathrm{hkl}}$. Estos valores son conocidos y tabulados en una base de datos. De este modo, mediante los valores obtenidos en la experiencia de difracción, y consultando la base de datos, podemos identificar el mineral. Puede ocurrir que dos minerales distintos posean parámetros de celda casi idénticos, en cuyo caso los espaciados $\mathrm{d}_{\mathrm{hkl}}$ serán casi iguales. En estos casos, hay que tener en cuenta otro resultado de la difracción: la intensidad con la que cada plano reticular refleja los rayos X. La intensidad depende de los átomos de la celda, y de su distribución. En resumen, los espaciados reticulares de los minerales dependen de los parámetros de la red, mientras que la intensidad de los rayos X reflejados por los planos reticulares depende de la estructura. La identificación de minerales mediante difracción utiliza, en primera instancia, los valores de los espaciados y, en segunda instancia, las intensidades reflejadas.

Así, en teoría, sin entrar todavía en la descripción de 
los dispositivos instrumentales, el método de identificación consiste en: a) Obtener los ángulos $\theta$ de reflexiones; b) Medir la intensidad reflejada y c) calcular, mediante la ley de Bragg, los espaciados reticulares. La lista de los espaciados, ordenados en orden decreciente (por lo tanto en orden creciente del ángulo $\theta$ ), con la correspondiente intensidad reflejada es la base de la identificación de minerales mediante difracción.

El método de polvo cristalino permite obtener una lista de espaciados e intensidades (en adelante datos de difracción) de la forma más rápida y sencilla. La muestra de mineral o de roca a identificar se pulveriza cuidadosamente, y el polvo se dispone en un portamuestras (Figura 1). En general, algunos $\mathrm{mm}^{3}$ son suficientes. El resultado de la pulverización es la obtención de un número muy elevado de pequeños cristalitos, por ejemplo $10^{9}$ cristalitos. Cada cristalito puede reflejar los rayos X si está en la orientación adecuada respecto del haz de rayos X. El número tan elevado de cristalitos en la muestra garantiza que todos los planos reticulares tendrán ocasión de producir reflexiones de Bragg, porque, para cada plano reticular $\mathrm{d}_{\mathrm{hkl}}$ siempre habrá un cierto número de cristalitos en la orientación adecuada respecto del haz de rayos X. Típicamente, el polvo puede disponerse formando una superficie plana de 1-2 $\mathrm{cm}^{2}$, o alternativamente, llenando un capilar de vidrio no absorbente, con un diámetro interior de unos $0.5 \mathrm{~mm}$.

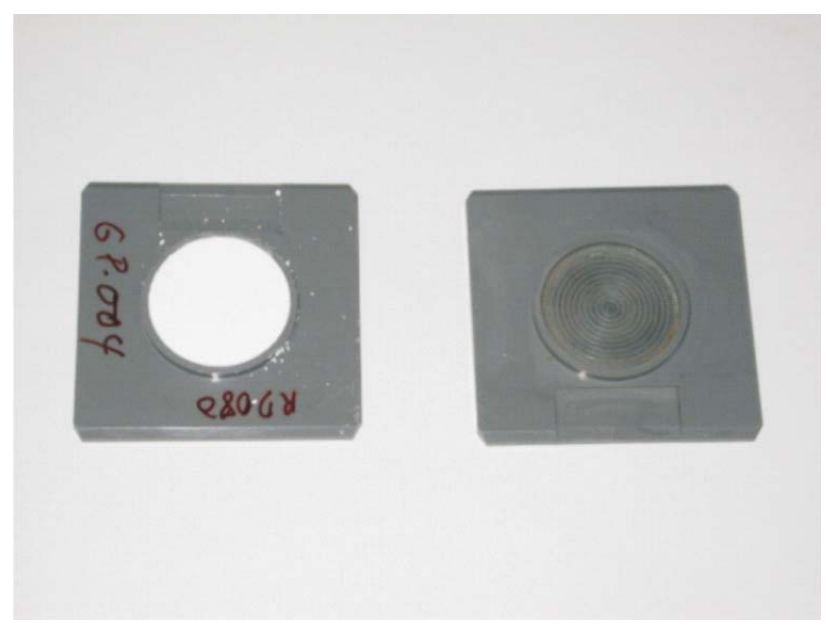

Figura 1. Portamuestras para polvo en difracción de rayos X, con y sin polvo cristalino.

El instrumento para la medida del difractograma es el difractómetro de polvo. Consiste en una fuente productora de rayos $\mathrm{X}$, un portamuestras montado sobre un goniómetro giratorio y un detector (Figura 2). El difractograma es un gráfico que en abscisas indica el ángulo de difracción y en ordenadas la intensidad correspondiente. Los máximos o picos de intensidad corresponden a reflexiones de Bragg.
Obtenido el difractograma, y extraídos los datos de difracción de la muestra problema, la identificación consiste en hallar en la base de datos el mineral cuyo difractograma corresponde con el problema. Puesto que la base de datos de difracción de polvo consta de muchos miles de entradas, es preciso utilizar un método sistemático que conduzca con seguridad a la solución del problema. Desde 1936 existe un método manual de identificación (Método Hanawalt), muy eficiente. Sin embargo, actualmente, la mayoría de laboratorios utilizan la identificación automática mediante potentes productos de software que son proporcionados por los mismos proveedores de equipos de difracción. Sea la identificación manual o automática, es necesario disponer de una base de datos de difracción. Aunque cada operador puede construir su propia base de datos, es más sencillo suscribirse a la base de datos del International Center for Diffraction Data (ICDD) que proporciona la base de datos en soporte digital o en papel (por ejemplo: Hanawalt Search Manual, Inorganic Phases; Figura 3).

Es evidente que la muestra problema puede ser una mezcla de dos, tres o muchos minerales. En estos casos, el difractograma experimental (difractograma multifásico) es la superposición de los difractogramas individuales de cada mineral por separado (Figura 4). Por lo tanto, en una mezcla compleja de muchos minerales, el difractograma presentará igualmente una complejidad grande. La identificación, en estos casos, requerirá del buen sentido y la experiencia del usuario del método. En el difractograma multifásico existe la posibilidad de cuantificar el porcentaje de cada mineral en la mezcla: en efecto, el mineral más abundante producirá intensidades de difracción mayores y la altura relativa de los picos de difracción permite obtener la cantidad de cada fase. En este método se pueden detectar minerales con menos de $1 \%$ en peso, pero en algunos casos especiales que permiten separación (minerales densos, minerales magnéticos) el límite de detección sobre la mezcla original (antes de proceder a la separación) puede ser mucho menor (por ejemplo $0.01 \%$ ). El tiempo total en un análisis de rutina, usando equipos modernos e identificación automática es inferior a los 30 minutos. Además, los equipos modernos realizan las medidas en serie de forma automática, usando un portamuestras de 15, $20 \mathrm{o}$ más muestras.

La difracción de polvo ha sido ampliamente utilizada, a nivel cualitativo, para identificar minerales en una mezcla. No obstante, en los últimos años se han mejorado sustancialmente las aplicaciones cuantitativas a partir del método de Rietveld (véase para una revisión del método, por ejemplo, Esteve, 2006). Esta poderosa herramienta, con el apoyo de software adecuado, posibilita el refinamiento de estructuras cristalinas a partir del diagrama de polvo. Pero, además, si se conocen los datos estructurales de los minerales de una mezcla, se pueden llegar a establecer las composiciones químicas de los componentes (en el caso de que cada uno de ellos forme parte de una serie isomórfica) y las proporciones de cada uno de los minerales en la mezcla. 

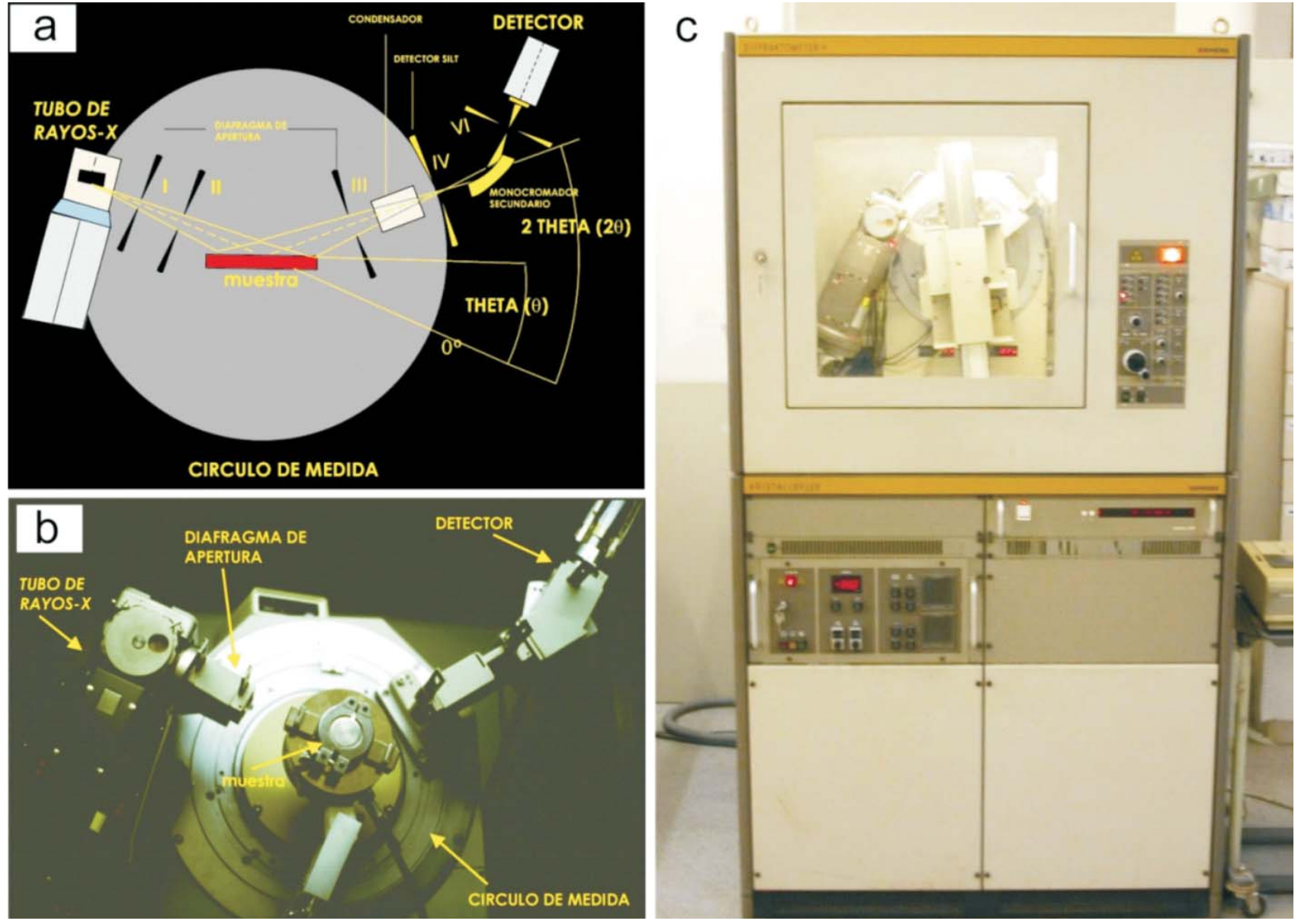

Figura 2. Difracción de polvo de rayos X. a) Geometría del difractómetro de polvo; b) detalle del difractómetro de polvo, que lleva acoplada una cámara de temperatura que permite realizar mediciones sobre la muestra expuesta a diferentes temperaturas, comparar con la imagen anterior; c) vista general del difractómetro de polvo Siemens D-500. Nótese el cargador automático de muestras. Serveis Científico-Tècnics, Universitat de Barcelona.

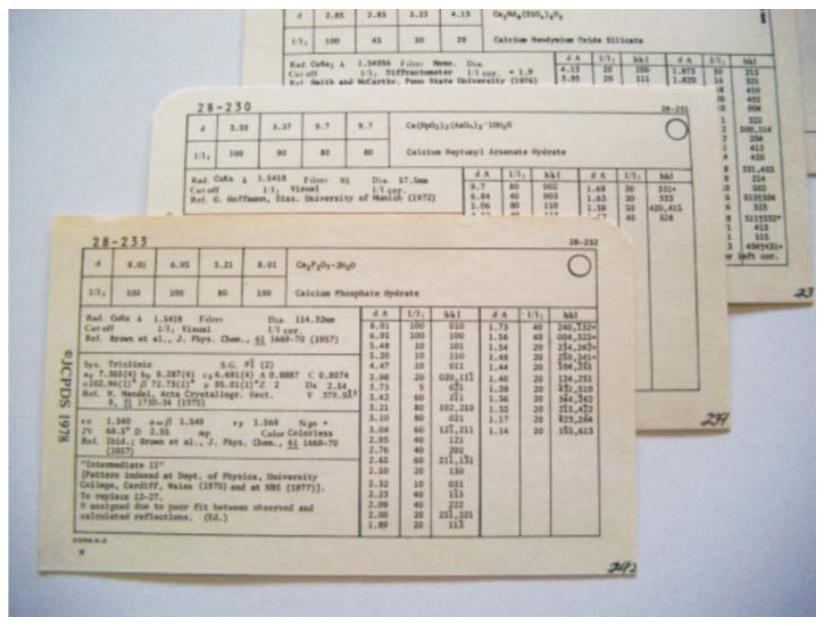

Figura 3. Fichas de la base de datos del ICCD. Existen una o varias fichas para cada mineral conocido. Actualmente esta información se halla en soporte digital.
Este método, por consiguiente, ofrece unas posibilidades enormes para la caracterización de fases en mezclas criptocristalinas. Evidentemente, ello incluye suelos, lateritas, arenas, concentrados de batea, escombreras de minas, material extraído de sondeos, etc. Desafortunadamente, el método no es todavía muy aplicado en minería, siendo por tanto un método con enormes capacidades de expansión a la resolución de problemas en este campo.

Como conclusión, podemos afirmar que el método de difracción de polvo es un método muy eficiente de identificación de minerales, individuales o mezclados.

\section{Microscopio electrónico de barrido con analizador de energías (SEM/ESEM-EDS)}

El microscopio electrónico de barrido (scanning electron microscope, SEM) se basa en la obtención de una imagen de la muestra a partir del barrido de la misma con un haz de electrones, como resultado de las interacciones entre los electrones incidentes y la muestra. El SEM se compone de varios elementos básicos: un cañón de 


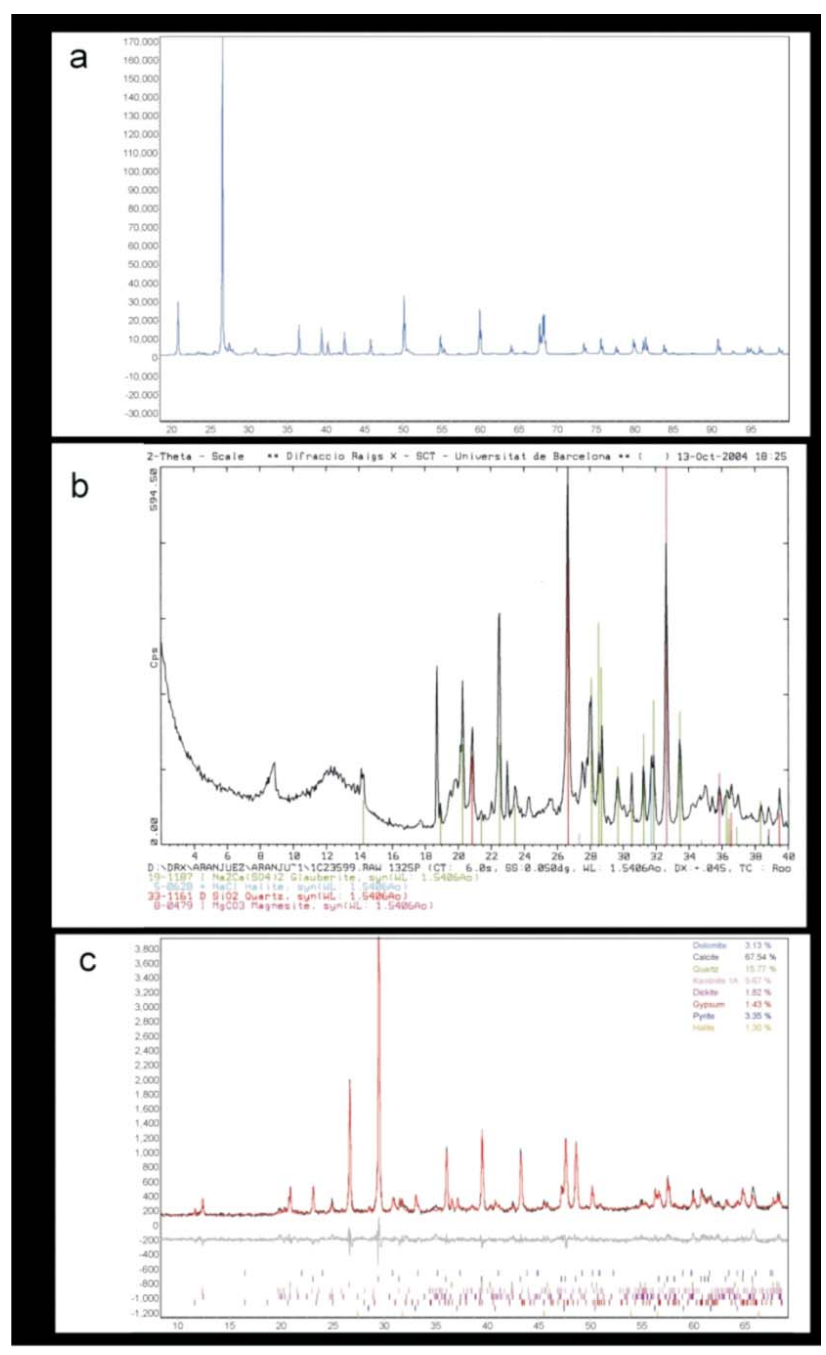

Figura 4. Procesado de espectros de polvo de rayos X. a) Difractograma sin identificar. El eje de abscisas indica el ángulo de Bragg. El eje de ordenadas es la intensidad (fotones por segundo); b) Difractograma con las fases presentes identificadas mediante software especializado; c) Difractograma con las fases identificadas y cuantificadas mediante análisis de perfil.

electrones con un filamento emisor de electrones, lentes magnéticas que dirigen y focalizan el haz de electrones sobre la muestra, sistema de barrido, portamuestras móvil y con giro universal, sistemas de obtención de la imagen y de análisis (Figura 5). Para analizar una muestra en el SEM se requieren generalmente condiciones estrictas de vacío en el interior del microscopio, ya que de lo contrario los electrones pueden ser dispersados por las moléculas de aire. Además, los mejores resultados se obtienen con muestras conductoras o convertidas en conductoras mediante un recubrimiento pelicular con un material conductor (generalmente, grafito; pueden emplearse también oro o aluminio). No obstante, actualmente existen microscopios electrónicos que no precisan ni recubrimiento de la muestra ni alto vacío en la cámara. Son los denominados microscopios electrónicos ambientales (Environmental
Scanning Electron Microscope, ESEM).

Las interacciones entre los electrones incidentes y la muestra originan la emisión de electrones secundarios, de electrones retrodispersados (Figura 6) y de rayos X característicos de los elementos presentes en la muestra (para el análisis químico cualitativo y, en algunos casos, semicuantitativo). En el SEM, diferentes detectores amplifican la señal emitida por la superficie de la muestra cuando es barrida por un delgado haz de electrones. La intensidad de la señal amplificada es visualizada en una pantalla de televisión convencional.

Las interacciones entre los electrones incidentes y los átomos de la muestra se clasifican en elásticas, inelásticas y emisión de radiación de frenado. Las colisiones elásticas modifican la trayectoria de los electrones incidentes, mientras que las colisiones inelásticas provocan una pérdida de energía. Los electrones secundarios (secondary electrons, SE) son electrones de la muestra que son emitidos durante las colisiones inelásticas (Figura 6). En cambio, los electrones retrodispersados (backscattered electrons, BSE) son aquellos electrones del haz incidente que son reflejados por la muestra tras sufrir múltiples colisiones elásticas e inelásticas (Figura 6).

Los electrones secundarios tienen, por convenio, una energía menor de $50 \mathrm{eV}$. El número de electrones secundarios que se produce durante el bombardeo de la muestra varía con el ángulo de incidencia del haz sobre la muestra, pero, en cambio, el número atómico promedio de los elementos presentes en la muestra tiene poca influencia (Figura 7). En cambio, el número de electrones retrodispersados aumenta casi linealmente con el número atómico Z (Figura 7).

Por consiguiente, si la muestra es rugosa, cada sector de la muestra enviará hacia los detectores una diferente cantidad de electrones secundarios dependiendo de la inclinación de cada sector; en la pantalla, se apreciarán unas zonas iluminadas (correspondientes a zonas que producen muchos electrones secundarios), otras zonas de sombra (zonas que producen pocos electrones secundarios) y zonas con diferentes claroscuros (con diversas producciones intermedias de electrones secundarios). Es precisamente esta asociación de zonas iluminadas, oscuras y claroscuras lo que da una sensación de relieve (figura 8). Por consiguiente, los electrones secundarios son ideales para el estudio morfológico de las muestras, alcanzando una resolución que puede ser inferior a $1 \mathrm{~nm}$. Todo ello encuentra aplicación, por ejemplo, en el estudio de detalle de la morfología de granos de arena en un concentrado de batea o el de microcristales en suelos o zonas de alteración, y en el de minerales de las arcillas.

El número de electrones del haz incidente que son retrodispersados depende de la composición química promedio del mineral excitado. De este modo, cuanto más alto sea el promedio de los números atómicos de los elementos que componen el mineral, tanto mayor será el número de electrones retrodispersados. 


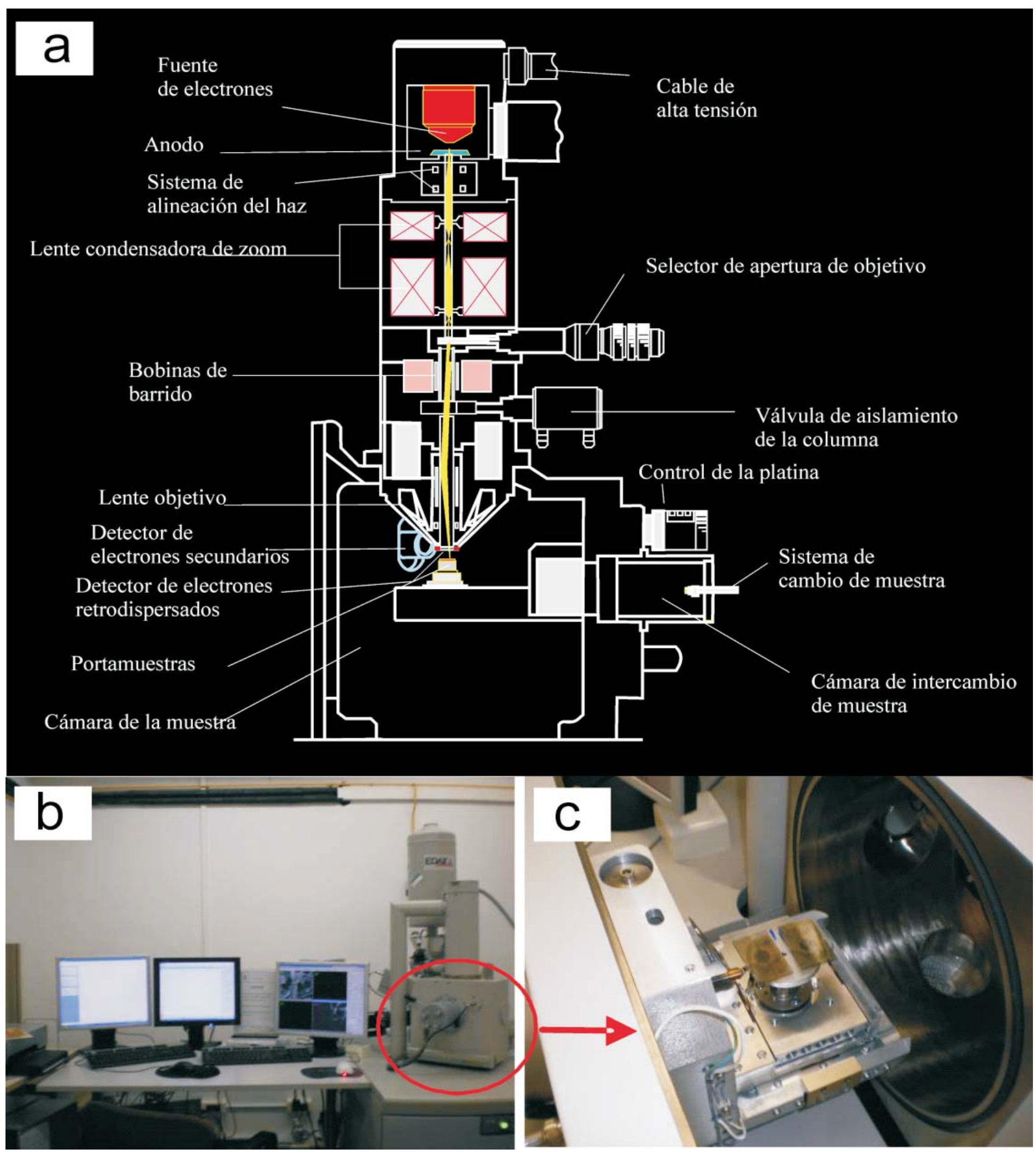

Figura 5. a) Estructura interna del SEM-EDS. b) Fotografía general del SEM (ESEM Quanta 200 FEI, XTE 325/D8395). C) Detalle de la introducción de una lámina delgada en el portamuestras.

Por consiguiente, los minerales pesados emiten más electrones retrodispersados que los ligeros, de modo que los detectores captan mucha más intensidad y, por tanto, transmiten a la pantalla una imagen brillante. Así pues, las áreas donde existan más elementos pesados se ven más brillantes y donde aparezcan elementos más ligeros, más oscuras. Por tanto, los detectores de electrones retrodisper- sados son muy útiles para obtener imágenes de los cambios composicionales (mapas de contraste de Z), obteniéndose los mejores resultados sobre muestras pulidas. Como aplicaciones de estos principios, por ejemplo, destaca la posibilidad de poner de manifiesto zonaciones intracristalinas, incluso aquéllas que puedan ser progresivas; también son muy útiles para obtener secuencias de cristalización a 


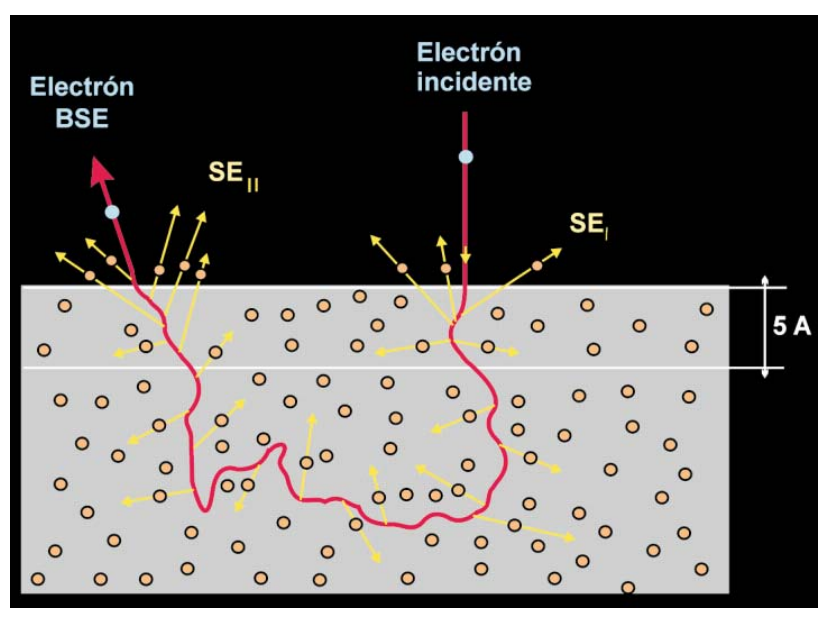

Figura 6. Interacción de los electrones incidentes con los átomos de la muestra y producción de electrones secundarios. Nótese que sólo pueden escapar los electrones secundarios generados durante la entrada de los electrones incidentes (SEI) o durante su salida (SEII) en los primeros nanómetros por debajo de la superficie, el resto es absorbido por la muestra. Los electrones incidentes tienen una trayectoria en forma de zig-zag dentro de la muestra y pueden acabar siendo reflejados (electrones retrodispersados) o absorbidos.

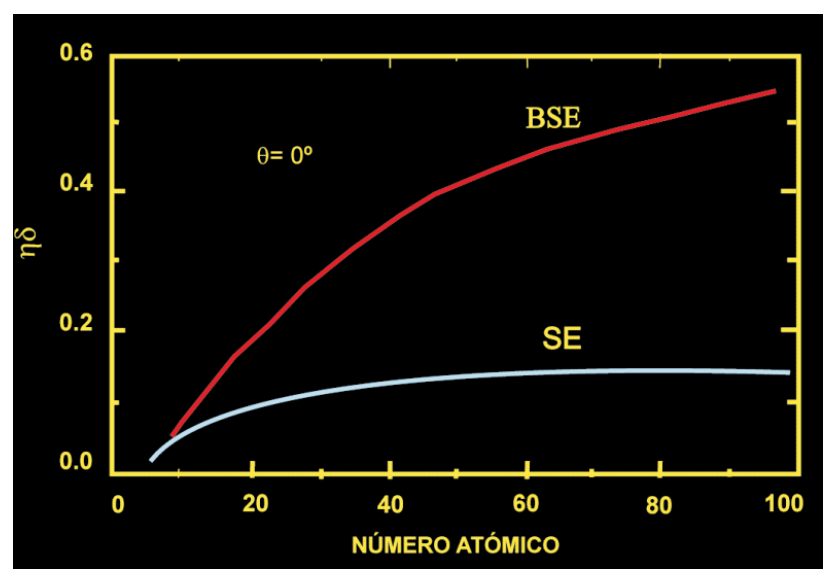

Figura 7. Comparación del número de electrones retrodispersados (BSE) y secundarios (SE) producidos en las mismas condiciones experimentales en función del número atómico de la muestra. Nótese el crecimiento casi lineal de los electrones retrodispersados al aumentar el número atómico.

microescala (Figura 9). Pero, sobre todo, las imágenes de electrones retrodispersados serán muy útiles para localizar minerales con elementos pesados, tanto en concentrados de batea como en muestras pulidas (Figura 10).

Por otra parte, durante las colisiones inelásticas, los electrones incidentes pueden arrancar electrones de las capas más profundas de los átomos, siempre y cuando la energía del electrón incidente sea superior al umbral de ionización de la capa atómica en cuestión. Cuando un átomo tiene una vacante en una capa interna, se produce un salto de un electrón de una capa superior para llenar dicha vacante, que dejará otra vacante en la capa superior. Ésta se llenará a su vez mediante un salto electrónico de una capa

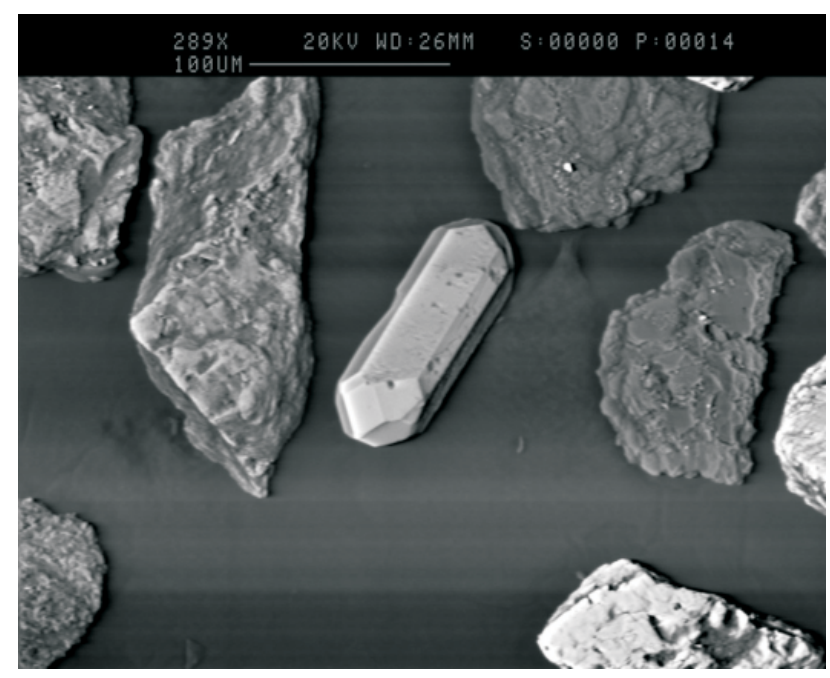

Figura 8. Morfología de granos de arena en un concentrado de batea. Entre diversos granos irregulares de cuarzo destaca un cristal de circón, idiomórfico y de hábito prismático. En este cristal la existencia de zonas más claras, oscuras y claroscuras da sensación de relieve.

superior y así sucesivamente. Durante cada salto, el átomo puede emitir rayos $\mathrm{X}$ característicos, que se denominan así ya que su energía es "característica" de cada elemento químico (la energía de un rayo X es igual a la diferencia de energías de los niveles atómicos involucrados). Como resultado del impacto electrónico, el átomo emite una familia de rayos $\mathrm{X}$ característicos.

Los microscopios electrónicos de barrido pueden incorporar un detector de de rayos X del tipo "dispersivo" en energía (Energy Dispersive Spectrometer, EDS) que permite identificar cuáles son las energías de los rayos X emitidos por la muestra $y$, por lo tanto, saber qué elementos químicos existen en la muestra. De este modo, al espectro continuo de rayos $\mathrm{X}$, originado por la radiación de frenado, se le superpone una serie de picos que se corresponden con las radiaciones características de cada uno de los elementos presentes en la muestra (Figura 11). Este espectro se genera en muy pocos segundos y la identificación del elemento que genera cada uno de los picos es inmediata, de modo que el análisis cualitativo de todos los elementos químicos (a partir del berilio) presentes en la muestra por encima del límite de detección del método puede realizarse en escasos segundos. Por tanto, este método es práctico (en tiempo y costo) para realizar análisis cualitativos puntuales. Puesto que el haz de electrones que excita la muestra puede ser muy fino (menos de $1 \mu \mathrm{m}$ ), el uso del EDS en el SEM permite identificar los elementos presentes en volúmenes de muestra inferiores a $1 \mu \mathrm{m} 3$; además, como se puede trabajar con corrientes de sonda muy bajas, el daño debido a la radiación es bajo y por tanto es un método poco destructivo y puede aplicarse al estudio de minerales lábiles, en particular, algunos minerales hidratados que aparecen como productos de alteración de balsas de residuos y que tienen implicaciones medioambientales. 

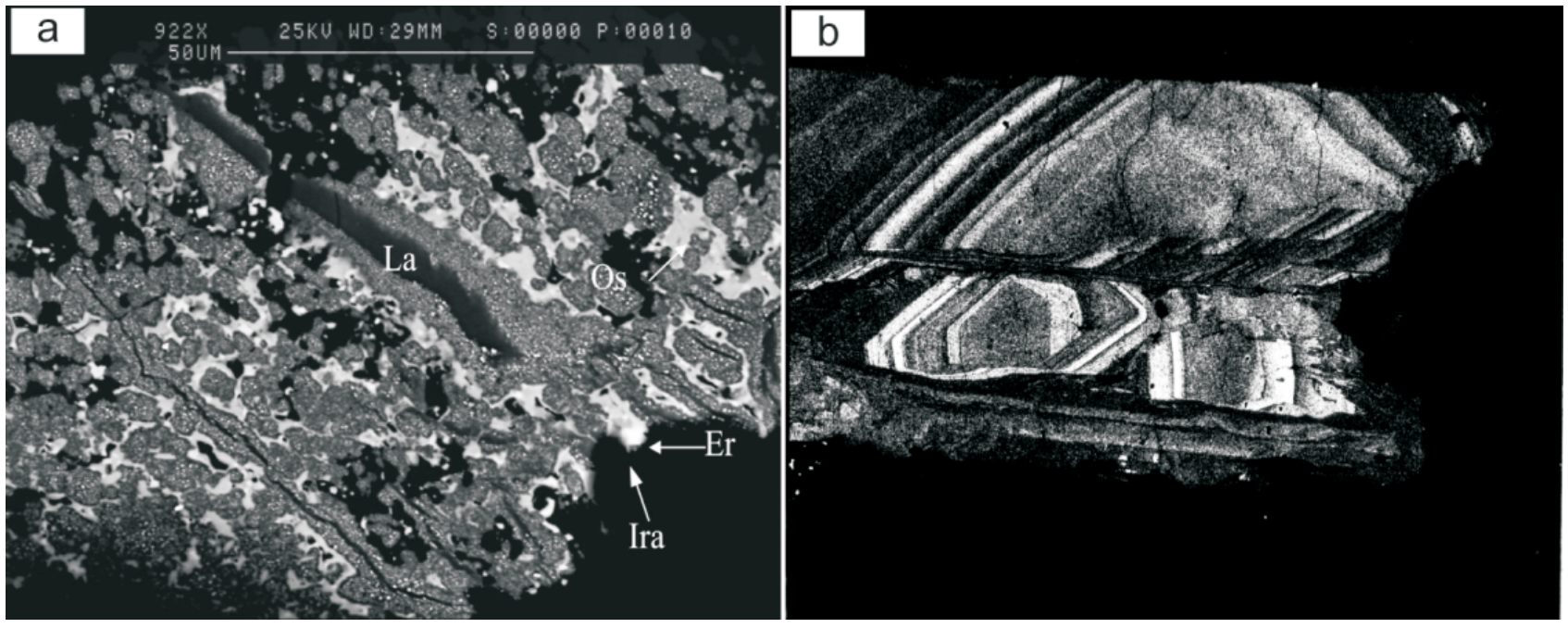

Figura 9. Imagen de electrones retrodispersados de minerales. A) Pepita tomada de un concentrado de batea, compuesta por diferentes minerales del grupo del platino. Nótense los intercrecimientos de laurita III (tono intermedios de gris) con irarsita (más brillante) que reemplaza a la laurita I (gris más claro). Las zonas más oscuras corresponden a zonas más ricas en rutenio (laurita II). Placer lateral de playa Mejías en Cuba Oriental (Díaz-Martínez et al., 1998). La=laurita, Ira=irarsita, Er= erlichmanita. B) Zonación compleja en cristales de la serie ferrocolumbita-tantalita de las pegmatitas del Cap de Creus (Catalunya). Las zonas más claras son más ricas en Ta (elemento más pesado), las más oscuras, en Nb. Nótese el paso progresivo entre algunas de las zonas. Este tipo de imágenes pueden ser imprescindibles a la hora de diseñar el beneficio de las menas.
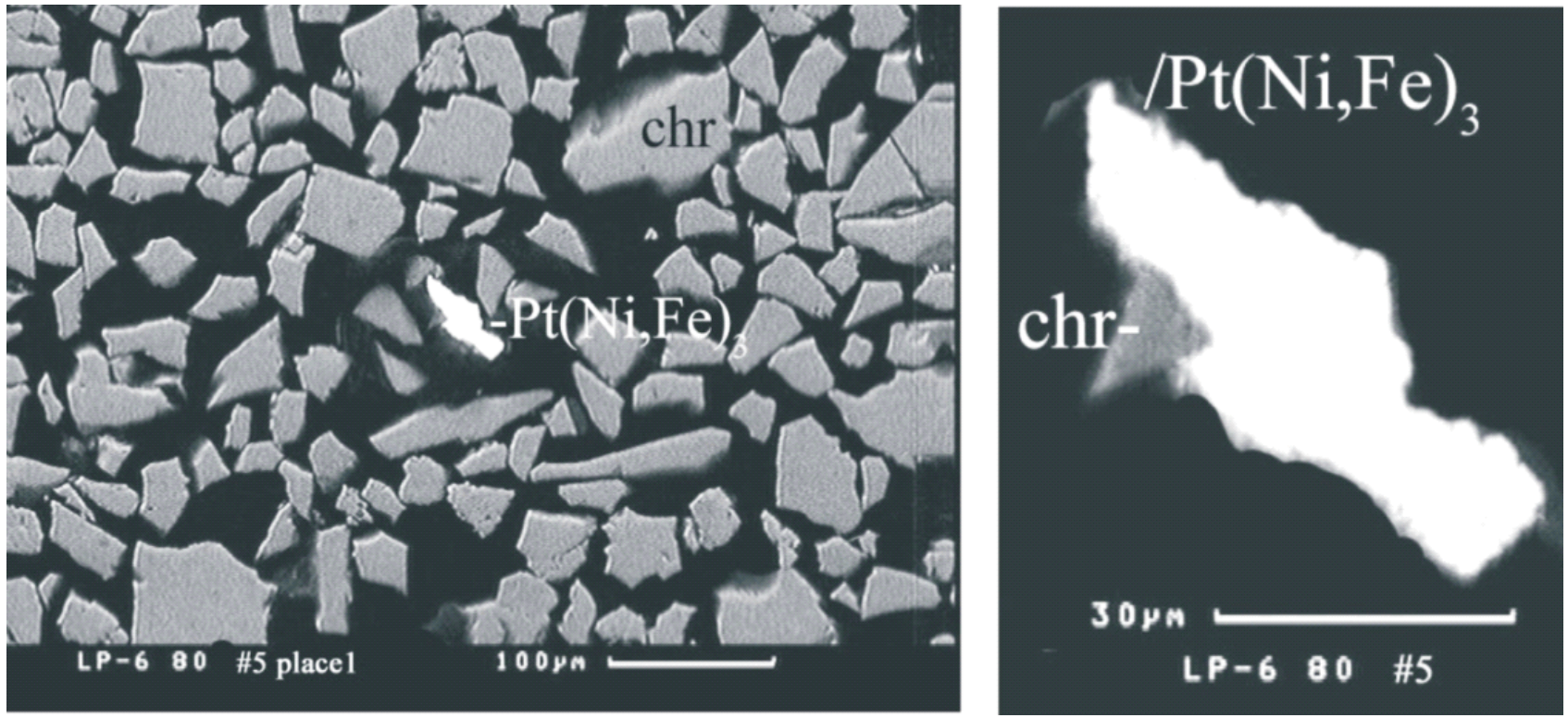

Figura 10. Imagen de electrones retrodispersados de un concentrado pesado, obtenido mediante el método de hidroseparación, de una muestra de cromitita $(\sim 3 \mathrm{~kg}$ ) de Loma Peguera (Cordillera Central de la República Dominicana). Nótese la mayor reflectividad electrónica de la aleación de Pt-Ni-Fe con respecto a los granos de cromita. Este tipo de imágenes son muy útiles en prospección.

Por otra parte, la intensidad de los rayos X producidos por cada elemento depende de la cantidad del mismo que haya en la muestra, de modo que el espectro en energía de los rayos X emitidos (intensidad versus energía) contiene dos niveles de información: por una parte, al espectro continuo se le superpone el espectro característico, en el que la posición de cada pico indica la energía de una radiación $\mathrm{X}$ característica de un elemento; por otra parte, la intensidad de cada pico (o lo que es lo mismo, la altura del mismo), es directamente proporcional a la cantidad de este elemento en la muestra. Por tanto, puede utilizarse este método para determinar la composición química de un volumen de muestra que, si lo deseamos, puede ser puntual, del orden de $1 \mu \mathrm{m}^{3}$. 


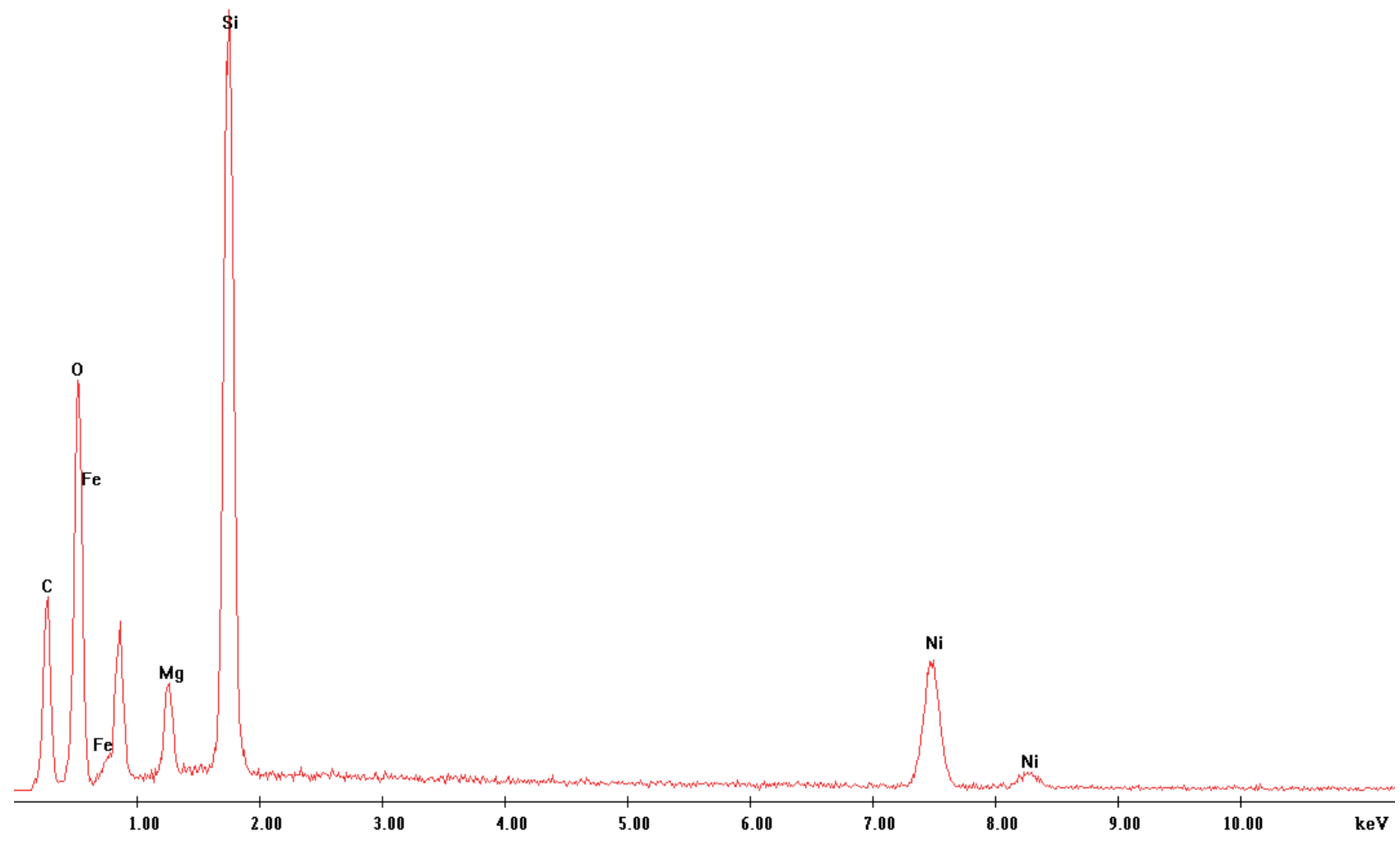

Figura 11. Espectro de rayos X obtenido por SEM-EDS de una muestra de garnierita en el que se aprecia el espectro característico de sus componentes $(\mathrm{Si}, \mathrm{O}, \mathrm{Ni}, \mathrm{Mg}, \mathrm{Fe}$ ) superpuesto al espectro continuo (fondo casi plano). El pico de $\mathrm{C}$ presente en el espectro proviene del recubrimiento de carbono de la muestra, que se le da para conferirle conductividad eléctrica. En este estudio podemos discriminar los componentes principales, pero también los accesorios.

Naturalmente, si se dispone de patrones adecuados con composición conocida, este análisis cualitativo se puede transformar en cuantitativo, de forma análoga a como se trabaja en cuantificación con la microsonda electrónica (véase apartado siguiente). También se puede utilizar un método sin patrones (denominado análisis "standardless"). Desgraciadamente, muchos elementos producen rayos $\mathrm{X}$ con energías próximas a las de otros elementos y debido a la baja resolución espectral de los espectrómetros EDS, los análisis obtenidos no siempre son muy precisos, pese al desarrollo reciente de software que ha mejorado los resultados. No obstante, debido a que puede trabajar con bajas corrientes de sonda, es un método muy poco destructivo, que lo convierte en imprescindible para el análisis semicuantitativo de cristales de tamaño de grano muy fino (menos de $1 \mu \mathrm{m}$ ), incluso hidratados, y en muestra rugosa. Por consiguiente, el microanálisis semicuantitativo mediante SEM-EDS es una herramienta potencialmente muy poderosa y posiblemente infrautilizada actualmente en el campo de las Ciencias de la Tierra.

\section{Análisis mediante catodoluminiscencia (CL)}

Aunque la catodoluminiscencia es una herramienta muchísimo más utilizada en exploración de cuencas petro- líferas, su potencial en la exploración minera es importante $\mathrm{y}$ no debe ser pasado por alto.

\subsection{Fundamentos del método de la catodoluminiscencia}

La catodoluminiscencia es un caso particular de luminiscencia en que la fuente de excitación son electrones. La catodoluminiscencia se produce sólo en el caso de que la estructura cristalina afectada presente algún tipo de defecto, entre los que cabe citar alguno de los siguientes: a) noestequiometría, b) imperfecciones estructurales (desorden, destrucción por radiación, destrucción por impacto), c) impurezas substitucionales o intersticiales que distorsionen la red cristalina. Es común que los cristales naturales presenten defectos, que actuarán como zonas en que se absorbe de forma preferente la energía del haz de electrones. De este modo, los dominios de imperfección se convierten en centros de luminiscencia.

La intensidad de la catodoluminiscencia es función de la densidad de corriente sobre la muestra y el voltaje (potencial de aceleración) de la corriente de sonda aplicada. Coy-YII (1970) demostró que la intensidad de la catodoluminiscencia varía en función de la corriente electrónica aplicada y del mineral: a) la catodoluminiscencia aumenta de forma no lineal con la intensidad de corriente, pero 
cuando se alcanza una determinada intensidad de corriente electrónica (nivel de saturación), típica de cada mineral, deja de incrementar la intensidad de la catodoluminiscencia; b) el aumento de la intensidad por encima de este nivel conlleva la disminución de la catodoluminiscencia (fase de inhibición).

El equipo de catodoluminiscencia (Figura 12a) puede ser montado en un microscopio electrónico o en un microscopio óptico (Figura 12b). En este caso, se pueden complementar los datos texturales obtenidos con microscopía óptica de luz transmitida y/o reflejada con los de catodoluminiscencia. Hay dos tipos de equipo de catodoluminiscencia: de cátodo "frío" (10-20 keV) y de cátodo "caliente" (25-30 keV). Las catodoluminiscencias trabajan normalmente a $10-20 \mathrm{keV}$ y a 1-5 mA de corriente. No obstante, la catodoluminiscencia de algunos minerales significativos, como el cuarzo, sólo es apreciable con cátodo "caliente". Es preciso respetar las normas de trabajo para cada equipo, pues se corre el riesgo de que en condiciones extremas se generen rayos-X que pueden ser peligrosos para la salud de los investigadores.

Como en todos estos casos, se trabaja con láminas no cubiertas. Es preciso evitar los cementos tipo bálsamo del Canadá y Lakeside, que pueden evaporar y, por tanto, romper la muestra y contaminar el equipo de vacío. Algunas resinas epoxy dan catodoluminiscencia amarillenta. Aunque no es un requisito pulir la muestra, se obtienen mejores resultados con muestras pulidas.

En el caso de que el equipo trabaje con cátodo frío no se requiere ningún tratamiento previo de la muestra, aunque en caso de que se estudien carbonatos Ebers y Kopp (1979) proponen teñir primero la muestra con solución de ferrocianuro potásico. La catodoluminiscencia caliente, en cambio, precisa recubrimiento de la muestra. La muestra puede ser estudiada en una lámina delgada pulida.

\subsection{Aplicaciones de la catodoluminiscencia}

La catodoluminiscencia tiene sus principales aplicaciones en el estudio textural de la lámina, y puede reforzar las observaciones realizadas con microscopía óptica convencional. Los casos más típicos son los siguientes (Marshall, 1988):

i) Distribución de minerales en una muestra, en base a que cada mineral presenta una catodoluminiscencia diferente. El caso más utilizado es la discriminación de carbonatos, pero puede ser aplicado a la discriminación de otros minerales, entre ellos, los ricos en tierras raras.

ii) Apoyo para reconocer volúmenes pequeños de una fase mineral, como por ejemplo vetas finas, inclusiones, películas, etc. Este aspecto es muy importante a la hora de estudiar las propiedades mecánicas de una roca o agregado.

iii) Si existe en un mismo grano mineral una variación química en el elemento activador a lo largo del proceso de cristalización se pueden producir importantes variaciones en la catodoluminiscencia que ayudan a remarcar inhomogeneidades en el grano: zonaciones, sobrecrecimientos, reemplazamientos, exsoluciones, etc. Este aspecto es crítico para mostrar cambios en el ambiente deposicional del mineral, con todo lo que ello implica para la modelación del depósito. Por consiguiente, la catodoluminiscencia debería realizarse anteriormente a cualquier estudio geoquímico de detalle.

iv) El color de la catodoluminiscencia da una idea de la composición química del mineral. Aunque este aspecto no es cuantitativo, el elemento presente puede ser un indicador de un determinado proceso mineral y, por consiguiente, una guía para la exploración minera.

v) En agregados minerales, la existencia de diferentes generaciones de un mismo mineral puede ser diferenciada con ayuda de la catodoluminiscencia. Este aspecto es importantísimo para diferenciar diferentes generaciones de carbonatos (Figura 12c), un trabajo crítico en la exploración petrolera. Este aspecto puede ser también interesante en la modelación de procesos genéticos de gangas o menas de yacimientos minerales, en particular, en el estudio geoquímico.

\section{Microanálisis mediante microsonda electrónica (EMPA)}

La microsonda electrónica es el método más utilizado actualmente para el análisis químico puntual rutinario de minerales. La idea original del microanálisis utilizando los rayos $\mathrm{X}$ secundarios, generados por un haz de electrones focalizados sobre una muestra sólida pulida, fue de Raimond Castaing (Castaing y Guinier, 1950; McGee y Keil, 2001). Indiscutiblemente, la comunidad geológica está en una eterna deuda de gratitud con Raimon Castaing (véase revisión de McGee y Keil, 2001).

\subsection{Fundamentos de la microsonda electrónica}

En una primera aproximación, la microsonda electrónica (electron-microprobe analyzer, EMPA) se puede considerar como un microscopio electrónico de barrido que incluye unas unidades analizadoras adicionales, los espectrómetros dispersivos en longitud de onda (WDS, wavelength-dispersive spectrometer; Figura 13). La principal diferencia es que la EMPA está especialmente diseñada para el análisis cuantitativo, mientras que el SEM está diseñado para obtener imágenes de la muestra. Los espectrómetros WDS separan las radiaciones X características por su longitud de onda. Para ello, se utilizan cristales analizadores para separar las radiaciones $\mathrm{X}$ por medio de la ley de Bragg, detectores de radiación para medir los rayos $\mathrm{X}$ dispersados por los cristales, y se comparan las intensidades de rayos $\mathrm{X}$ emitidas por la muestra con las de patrones de composición conocida. 

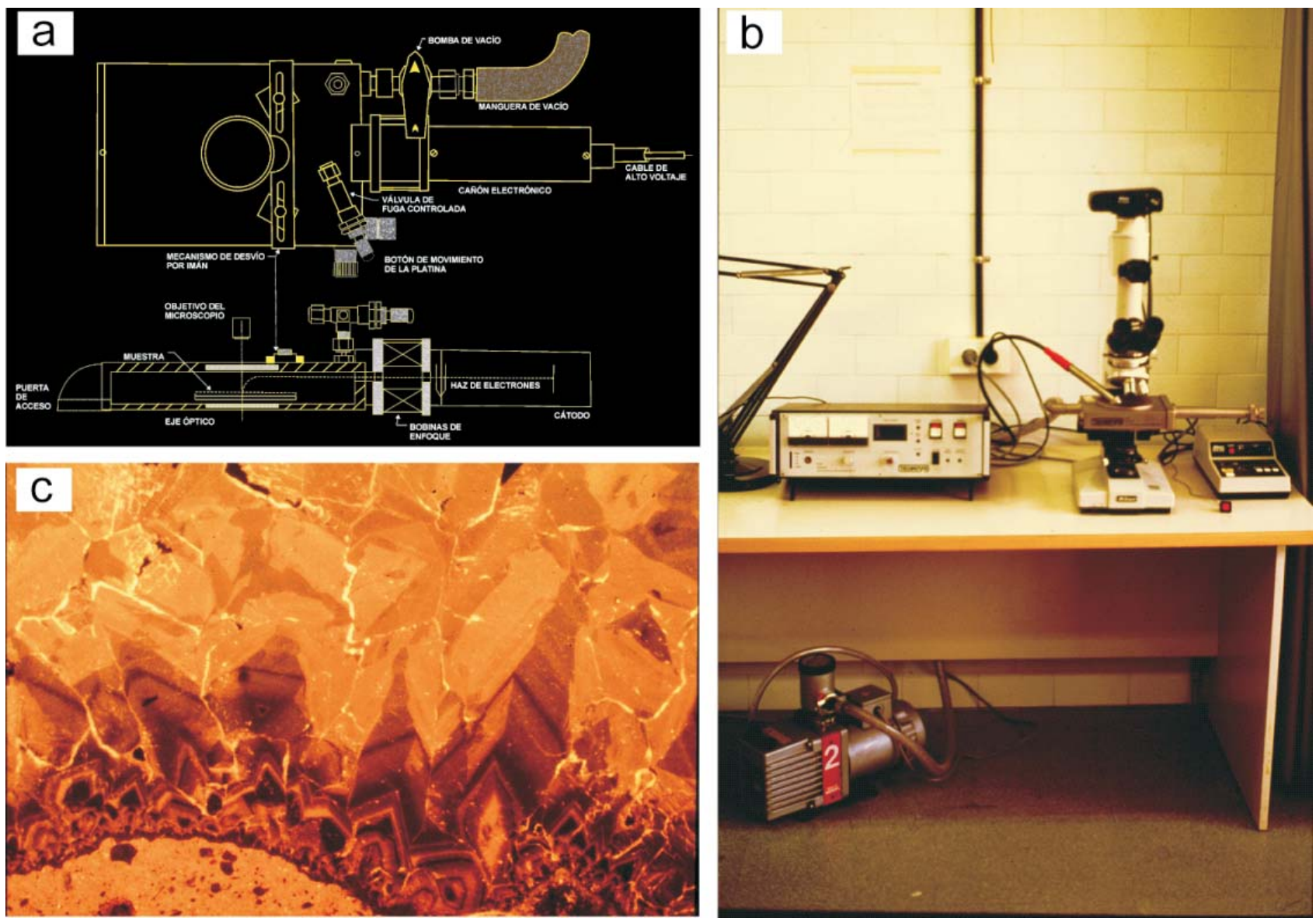

Figura 12. Funcionamiento de la catodoluminiscencia. A) Planta (arriba) y sección de un equipo de catodoluminiscencia. B) Equipo instalado sobre la platina de un microscopio; se observa también la bomba de vacío y los controles. C) Imagen de una muestra de calcita rica en manganeso en catodoluminiscencia. Se aprecian diferentes generaciones y las bandas de crecimiento. Estas imágenes son imprescindibles a la hora de plantear un estudio de inclusiones fluidas, isótopos o geoquímica.

Como en el microscopio electrónico, la muestra que se va a analizar (probeta o lámina delgada, que debe estar muy bien pulida y extremadamente limpia y seca) es previamente convertida en eléctricamente conductora, mediante un recubrimiento fino de algún material conductor. El material conductor es mayoritariamente grafito, pero también puede ser aluminio u oro. La muestra eléctricamente conductora es introducida en la cámara mediante un portamuestras, al vacío, y se busca la zona que se quiere analizar con la ayuda del microscopio óptico y de las imágenes de electrones secundarios y retrodispersados. Para ahorrar tiempo y dinero, las zonas en que se quiere obtener análisis cuantitativo deben estar adecuadamente marcadas y fotografiadas (a fin de localizar rápidamente los puntos deseados) y analizadas cualitativamente (a fin de programar la microsonda directamente para analizar los elementos necesarios).

De este modo, cuando tenemos localizado el punto donde queremos realizar el análisis, enfocamos el haz de electrones en el punto de interés y comienza el análisis propiamente dicho. A partir de este momento cada uno de los elementos que constituyen la sustancia que se encuentra en la superficie (hasta unas pocas micras de profundidad) responden emitiendo una familia de rayos $\mathrm{X}$ característicos. Esto implica que, mientras dura el impacto electrónico, la muestra emite continuamente diversas radiaciones $\mathrm{X}$ en todas las direcciones, cada una de las cuales tiene una energía (y por tanto una longitud de onda) característica de un elemento.

El problema que se plantea en este momento es el de analizar por separado las radiaciones X en un detector. Habitualmente se usan contadores proporcionales de rayos $\mathrm{X}$, que pueden determinar con mucha precisión la intensidad de rayos $\mathrm{X}$, y esta magnitud es proporcional a la cantidad de la substancia que está produciendo la radiación. El problema es que estos detectores no pueden discriminar entre las radiaciones de cada uno de los elementos. Por esto, se requiere separar las radiaciones de cada uno de los elementos de manera secuencial. Esta separación se realiza por medio de los cristales analizadores.

Los espectrómetros WDS se acoplan alrededor de la cámara. La microsonda puede contar con diversos espec- 

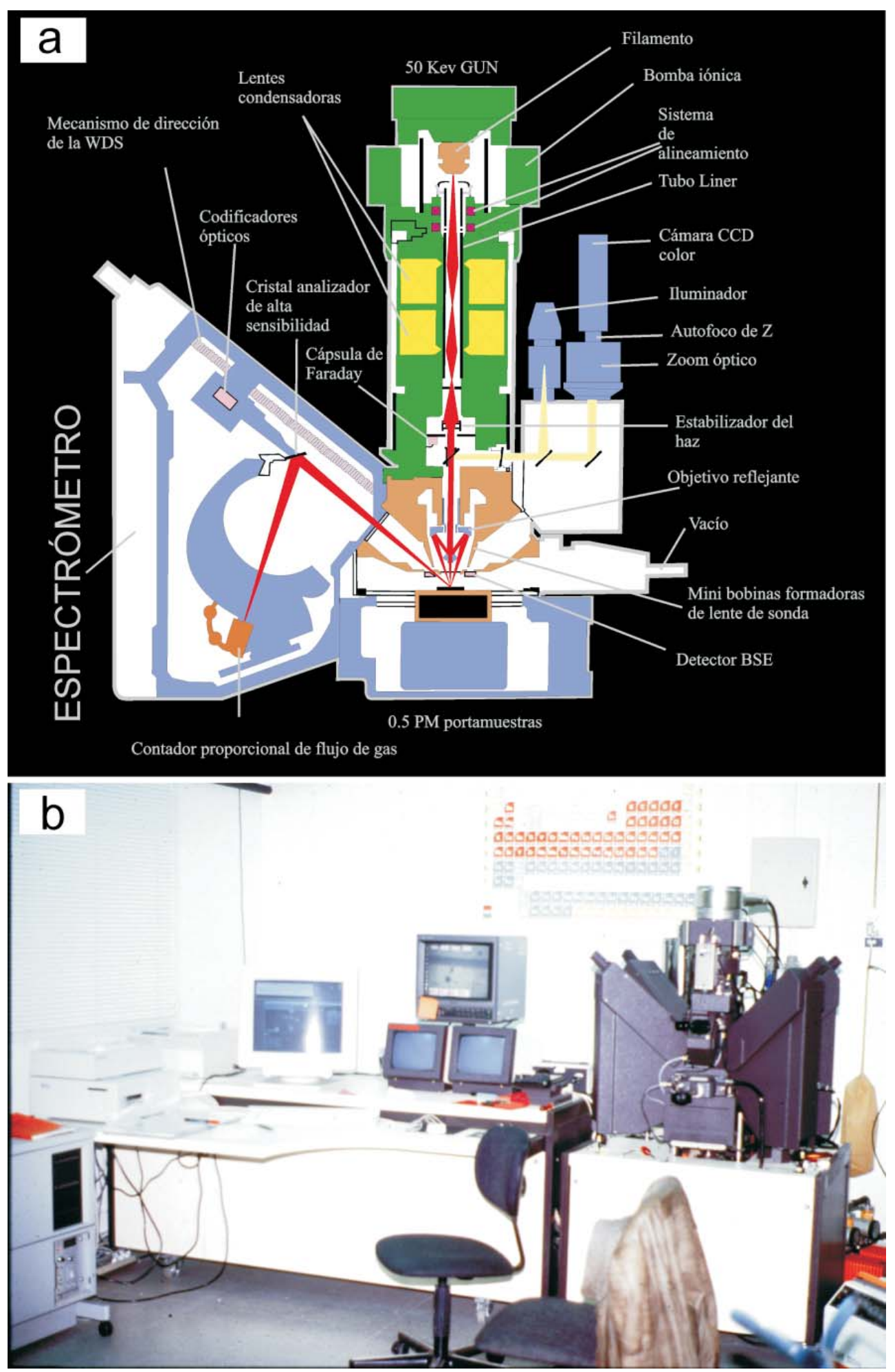

Figura 13. Ejemplos de equipos de microsonda electrónica. A) Diseño del equipo CAMECA SX 100. b) Equipo CAMECA SX50 instalado en los Serveis Científico-Tècnics de la Universitat de Barcelona, equipado con 4 espectrómetros WDS, 12 cristales y un EDS. A la izquierda, comandos y pantallas de control. 
trómetros, entre 1 y 5 . A grandes rasgos, cada espectrómetro consta de:

a) Una ventana estrecha que permite el paso al interior del espectrómetro de las radiaciones $\mathrm{X}$ que tienen el mismo ángulo de salida (take-off); por tanto, las radiaciones $\mathrm{X}$ de todos los elementos que están presentes en la muestra entran en el espectrómetro siguiendo todas ellas la misma trayectoria, pero cada una de ellas con su propia energía (o longitud de onda).

b) Uno o diversos cristales analizadores giratorios situados en la trayectoria de las radiaciones $\mathrm{X}$ y que utilizaremos para la separación de las radiaciones; de este cristal conocemos su estructura.

c) Uno o diversos contadores proporcionales que analizan y realizan el conteo de cada una de las radiaciones separadas por los cristales.

La separación de las diferentes radiaciones $\mathrm{X}$ se realiza de forma secuencial, aprovechando que cada una de ellas tiene una longitud de onda característica, a partir del fenómeno de la difracción de rayos $\mathrm{X}$ por redes cristalinas. Recordemos la ecuación de Bragg:

$$
\mathrm{n} \lambda=2 \mathrm{~d}_{\mathrm{hkl}} \sin \theta
$$

donde $\mathrm{n}$ representa el orden de difracción, $\lambda$ la longitud de onda de una radiación determinada, $\mathrm{d}_{\mathrm{hkl}}$ el espaciado reticular de un plano $\{\mathrm{hkl}\}$ de un cristal y $\theta$ el ángulo de incidencia.

Ésta es una ecuación con una sola solución para $\lambda$, en caso que consideremos $n=1$, posicionemos el cristal en un ángulo $\theta$ determinado y dispongamos de un cristal con un plano $\mathrm{d}_{\text {hkl }}$ determinado.

Por tanto, si queremos separar una radiación $\mathrm{X}$ determinada (R1) del resto, como en nuestro caso, se puede utilizar el fenómeno de la difracción por redes cristalinas (redes de difracción): podemos intercalar un cristal de espaciado reticular conocido en la trayectoria de los rayos $\mathrm{X}$ que emergen de la ventana. El ángulo que forma el plano $\mathrm{d}_{\text {hkl }}$ del cristal con la radiación lo podemos variar a nuestra conveniencia, de manera que podemos controlar qué radiación difracta y cuál no.

Este cristal se dispone de manera que la radiación que interese seleccionar incida con un ángulo $\theta$. De esta mane$\mathrm{ra}$, de todas las radiaciones $\mathrm{X}$ recibidas en el cristal analizador, el cristal sólo difracta hacia el detector la radiación cuya $\lambda$ cumple la ley de Bragg. El resto puede ir hacia otros posibles cristales analizadores que pueden estar posicionados para enviar cada una de las radiaciones seleccionadas hacia un detector.

El detector realiza el conteo de la radiación X que le llega de cada elemento en un tiempo determinado. Una vez cuantificada esta intensidad de la radiación recibida, la medición de este elemento queda terminada. A partir de este momento, se repite la operación para otro elemento, cambiando la orientación del cristal, el tipo de cristal, o ambas cosas. Esta operación se repite hasta que se han analizado todos los elementos de la muestra (Figura 14).

Cada detector mide las cuentas de radiación X que recibe en cada tanda. La intensidad de una determinada radiación $\mathrm{X}$ depende de la cantidad del elemento correspondiente que haya en la muestra. Para determinar la cantidad de un elemento determinado, se compara la intensidad producida por el mineral con la que generan un patrón de composición conocida, de acuerdo con la siguiente ecuación:

$$
\mathrm{I}_{\mathrm{m}} / \mathrm{I}=\mathrm{C}_{\mathrm{m}} / \mathrm{C}_{\mathrm{p}}\left[\mathrm{ZAF}\left(\mathrm{C}_{\mathrm{m}}\right)\right]
$$

donde $\mathrm{I}_{\mathrm{m}}$ es la intensidad de la radiación X generada por la muestra, $I_{p}$ es la intensidad de la radiación $X$ generada por el patrón de composición conocida, $\mathrm{C}_{\mathrm{m}}$ es la concentración del elemento problema en la muestra, $\mathrm{C}_{\mathrm{p}}$ es la concentración del mismo elemento en el patrón y ZAF es la denominada corrección por efecto de matriz. Ésta corrección no sería necesaria si muestra y patrón se comportaran de manera similar frente al haz de electrones. Como en la práctica no es así, es necesario corregir con factores que tengan en cuenta las diferencias de número atómico $(Z)$, absorción de rayos X (A) y fluorescencia (F) entre muestra y patrón. En ésta ecuación conocemos $\mathrm{I}_{\mathrm{m}}$ e $\mathrm{I}_{\mathrm{p}}$ (puesto que es la lectura que nos da el detector en cada caso) y $\mathrm{C}_{\mathrm{p}} \mathrm{y}$, por tanto, podemos obtener $\mathrm{C}_{\mathrm{m}}$, es decir, la concentración del elemento de interés. Ya que las correcciones ZAF dependen de la propia concentración $\mathrm{C}_{\mathrm{m}}$ ésta se debe obtener mediante un proceso iterativo. Obviamente, si se utiliza un patrón de composición similar a la de la muestra las correcciones ZAF serán mínimas.

\subsection{Aplicaciones de la microsonda electrónica}

Excelentes revisiones sobre la aplicación de EMPA a las ciencias geológicas se pueden encontrar en McGee y Keil (2001), Bowles (2002) y Reed (2005). La posibilidad de obtener análisis químicos cuantitativos de minerales permite el estudio de muchos problemas geológicos. Por ejemplo, establecer las condiciones de temperatura y presión de los minerales, o de las rocas en que ellos se encuentran. Las principales aplicaciones de EMPA a las ciencias geológicas son:

\subsubsection{Análisis químico cuantitativo puntual de elementos mayores}

La microsonda electrónica permite el análisis químico cuantitativo de volúmenes muy pequeños (del orden de 1 $\mu \mathrm{m}^{3}$ ), lo que posibilita el análisis puntual sistemático de granos heterogéneos (zonados, exsoluciones). Los elementos que podemos analizar con este proceso son los de número atómico mayor que 3 (a partir del berilio, inclu- 

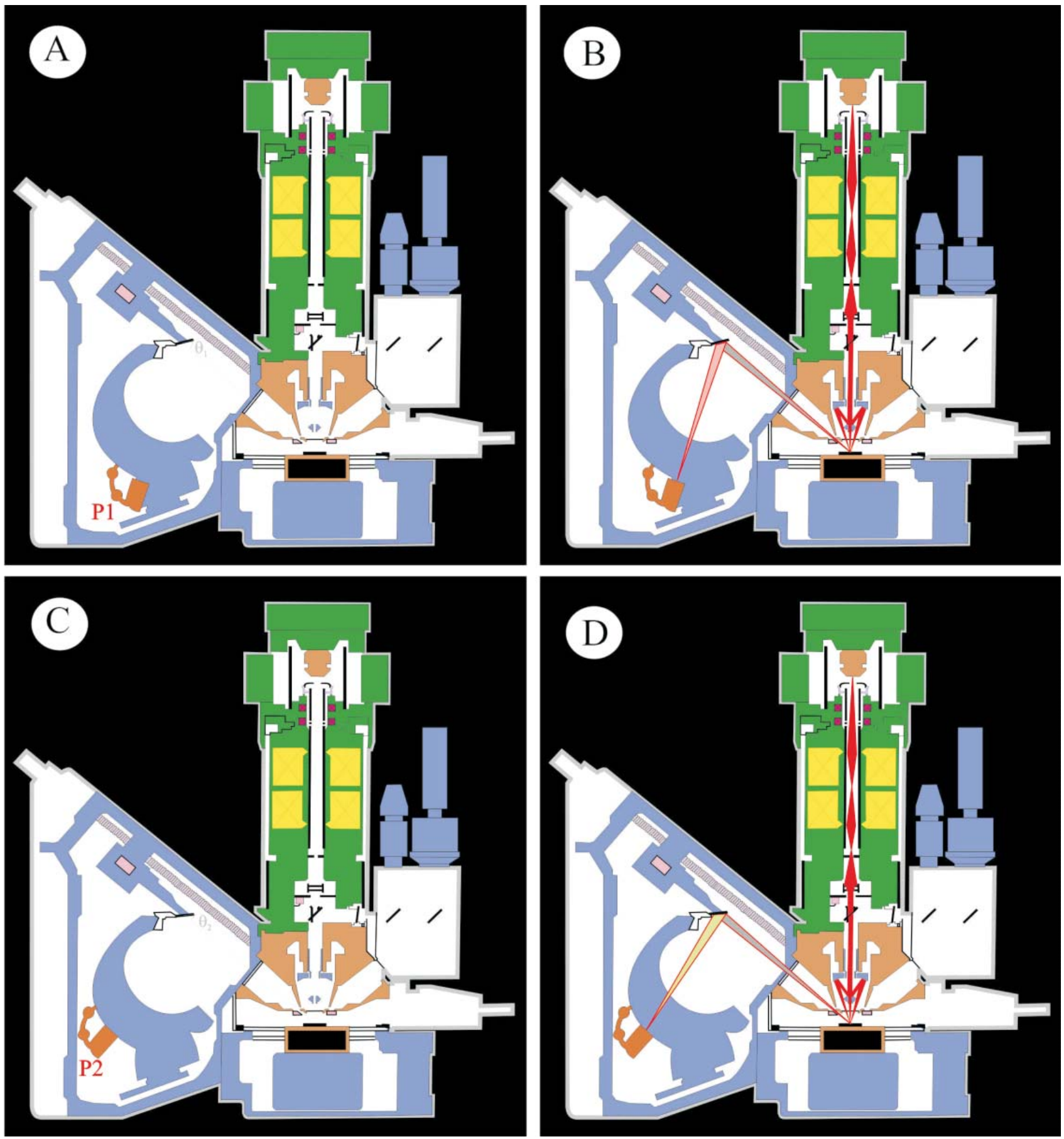

Figura 14. Análisis secuencial en la microsonda electrónica. A) Se localiza el punto de análisis adecuado para analizar un elemento determinado E1. Se sitúa el cristal analizador en la trayectoria de los rayos X y se gira de modo que difracte sólo la radiación X seleccionada como característica del elemento deseado; a la vez, se coloca el detector en la posición P1 para captar la radiación difractada con el ángulo $\theta$ adecuado. B) Comienza el análisis con esta geometría y se mide durante un tiempo determinado la radiación que llega al detector. C) Se prepara la microsonda para el análisis del siguiente elemento. Para ello se gira el cristal a una nueva posición para que difracte solamente la radiación X del nuevo elemento seleccionado, y se coloca en la posición apropiada (P2) el detector. D) En el detector se realiza la medición de la intensidad de la radiación X causada por el nuevo elemento, durante un tiempo determinado. Esta operación se repite las veces que sea necesario hasta que todos los elementos previstos han sido analizados.

sive) hasta el uranio (con microsondas blindadas podemos analizar también elementos actínidos con $\mathrm{Z}$ mayor que el uranio). Por desgracia, no se pueden analizar litio o helio, y mucho menos aún, hidrógeno, cosa que implica que el análisis de minerales hidratados tiene más dificultades. En cuanto a los límites de detección, dependen de cada elemento y especialmente de las condiciones de análisis (energía y corriente del haz de electrones, tiempo de con- 
teo, etc.), pero de forma rutinaria se trabaja bien hasta las centésimas de \%; llegar al nivel de partes por millón (ppm, $1 / 1000.000$ ) es posible, pero complicado, y no es una cosa rutinaria.

En cualquier caso, la microsonda permite conocer la composición química de un mineral, generalmente con una precisión relativa del $2 \%$, y por tanto identificarlo. Ello es imprescindible en la caracterización de nuevos minerales y muy útil para la identificación de minerales de tamaño pequeño (de pocas micras), como es el caso de los minerales del grupo del platino. Pero evidentemente también es imprescindible el método para determinar la concentración de elementos valiosos en la estructura de un mineral, así como también la concentración de los elementos penalizantes.

A efectos de rutina de trabajo, durante el análisis químico de un mineral se pueden presentar diversos casos:

a) Se conoce el grupo mineral al que pertenece el mineral que hemos de analizar y necesitamos saber su composición química (por ejemplo, sabemos que es una plagioclasa y queremos saber la proporción de sus términos extremos albita y anortita). Este es el caso más senci1lo, y también el más rutinario. En este caso tenemos tres grandes posibilidades:

a1) El mineral no tiene oxígeno en su composición. En este caso, analizamos directamente los elementos posicionando los cristales donde difracten la radiación que interesa, obteniendo con ello la composición en $\%$ en peso y a partir de ello la fórmula estructural. El resultado se puede dar en forma de $\%$ en peso y de proporción atómica de cada elemento. En minerales de uso rutinario, la suma de los \% en peso debería estar comprendida entre 99.5 y 100.5 para que el análisis se considere aceptable; en caso de minerales de composición más compleja este criterio puede variar ampliamente.

a2) El mineral tiene oxígeno en su estructura. En este caso, el oxígeno no se analiza, porque cualquier sal oxigenada se puede descomponer en la suma de sus óxidos, por ejemplo: $\mathrm{CaSiO}_{3}=\mathrm{SiO}_{2}+\mathrm{CaO}$

Por tanto, en el caso del mineral anterior, sólo sería preciso analizar $\mathrm{Si} \mathrm{y} \mathrm{Ca}$, posicionando el cristal analizador a las posiciones en que difracte la radiación del Si y posteriormente la del $\mathrm{Ca}$. Una vez conocida la proporción en \% en peso, se puede calcular la proporción de átomos de cada elemento analizado, y con ello asignar los oxígenos necesarios para completar la estequiometría. El resultado se da en forma de \% en peso de los óxidos correspondientes y de proporción atómica de cada elemento. Nuevamente, la suma de los \% en peso debe estar comprendida entre 99.5 y 100.5 para que el análisis se considere válido.

a3) El mineral tiene en su estructura oxígeno y agua (o bien otros elementos como el litio, que no se pueden analizar). Si conocemos su fórmula ideal, con el análisis podremos extrapolar el contenido de agua y de litio por estequiometría. El resultado se da nuevamente en forma de
$\%$ en peso de los óxidos correspondientes y de proporción atómica de cada elemento. Los hidrógenos se calculan en forma de agua.

a4) El mineral tiene elementos con diferentes estados de valencia. Pese a que hay programas que permiten teóricamente analizar para un mismo elemento la proporción de cada uno de sus estadios de valencia (cosa que es especialmente importante en el caso del Fe, que generalmente está en forma de $\mathrm{Fe}^{2+}$ y $\mathrm{Fe}^{3+}$ ), en la práctica este cálculo se puede realizar, si se conoce la fórmula estructural del mineral, por estequiometría y balance de cargas. El resultado se da en forma de \% en peso de los óxidos correspondientes (desglosando los óxidos correspondientes a los diferentes estados de valencia) y de proporción atómica de cada elemento.

b) Se conocen los elementos que hay en un mineral con oxígenos, pero no el tipo de mineral. En este caso podemos obtener la proporción en peso ( $\%$ en peso) de todos los elementos presentes en la muestra. Si el análisis de los óxidos suma 100, seguramente se trata de un mineral anhidro y podremos calcular su formula estructural por estequiometría. Si se desconoce el tipo de mineral que se tiene, el cálculo de la fórmula estructural será más complicado en caso de que haya agua o elementos con diferentes estados de valencia. El resultado se da en forma de $\%$ en peso de los óxidos correspondientes y de proporción atómica de cada elemento.

c) Se desconoce qué elementos hay en la muestra y de qué mineral se trata. Esta situación es complicada y debe ser evitada en lo posible, porque necesitamos saber en qué posición colocaremos el cristal y el detector y qué patrones deberemos calibrar (ecuación 2). En este caso, se requiere efectuar previamente un análisis cualitativo con SEM-EDS o con la propia microsonda. El análisis de SEM-EDS es rápido, pues en pocos segundos tenemos el análisis cualitativo de la muestra (listado de elementos que la forman). El análisis cualitativo por dispersión de longitudes de onda es más lento, ya que se precisa girar los cristales (y simultáneamente los detectores) muy lentamente a fin de ver si el detector capta señal. Todo ello puede aumentar el tiempo de análisis y encarecerlo extraordinariamente.

\subsubsection{Determinación de elementos trazas}

También el EMPA permite la cuantificación de elementos menores y trazas, con un límite de detección entre 10 y $50 \mathrm{ppm}$. Sin embargo, bajo condiciones de análisis extraordinarias (p.e. corrientes de $8 \mathrm{~mA}$ y tiempos de conteo de 2000 s) para algunos elementos $(\mathrm{Cr}, \mathrm{Ti}, \mathrm{Si})$ se han llegado a alcanzar límites de detección a niveles de sub-ppm (Robinson et al., 1998). Ejemplos de esta aplicación son la determinación de elementos traza del grupo del platino en sulfuros (Gervilla et al., 2004) y la de $\mathrm{U}$, Th y $\mathrm{Pb}$ en monacita para investigaciones geocronológicas (Cocherie et al., 1998; Williams et al., 1999). 


\subsubsection{Mapas de distribución de elementos}

Se puede realizar un mapa de la distribución de un elemento en un grano, mediante el barrido del haz electrónico por todo el grano, manteniendo constante la posición angular del cristal analizador, de forma que sólo se registra la variación del contenido de un elemento a lo largo y ancho del grano. Esta operación puede realizarse simultáneamente para otros elementos utilizando otros espectrómetros disponibles en la microsonda.

Una vez realizado el mapa de uno o varios elementos, un nuevo barrido del grano nos puede dar el mapa de uno o varios elementos simultáneamente, y se procedería del mismo modo hasta completar el número de elementos que se considere necesario.

Estos análisis pueden ser complementados por microfotografía y pueden ser un argumento muy valioso para localizar a escala de grano elementos químicos de interés económico, o bien poner de manifiesto qué tipos de substituciones se dan en la estructura del cristal. En efecto, cuando aumenta la concentración de un elemento mientras que baja la de otro en paralelo, este fenómeno indica que los dos elementos se substituyen en la estructura del mineral; en otros casos, se pueden dar substituciones simultáneas de un elemento por la suma de otros (Figura 15).

\subsubsection{Perfiles de distribución de elementos}

Otra posibilidad es la de realizar un perfil de distribución de un elemento a lo largo de un grano o de una muestra. En este caso, el haz electrónico o alternativamente el portamuestras, hacen un recorrido en la dirección que se les indique y se posiciona el cristal analizador de forma que difracte hacia el detector sólo este elemento. Como resultado, en la imagen de microscopía electrónica se puede hacer superponer una gráfica que refleje la intensidad de la radiación emitida por el elemento en cada punto del perfil. Por tanto, si el cristal es homogéneo, la gráfica será plana, pero si presenta inhomogeneneidades presentará máximos (puntos con mayor proporción del elemento) y mínimos (puntos más pobres en este elemento). Se puede obtener un perfil cuantitativo, analizando puntos de la muestra sobre la línea, a intervalos regulares entre cada uno de los puntos. Evidentemente, si en la misma gráfica se comparan los datos de dos elementos que se sustituyen mutuamente en la estructura del mineral, en este caso los máximos de un elemento coincidirán con los mínimos del otro.

Un aspecto muy interesante es ver el comportamiento de algunos elementos en el contacto entre dos granos de minerales diferentes pero que tienen algunos elementos en común: la repartición de los elementos entre estos dos minerales se realizará en función de las condiciones del medio en que cristalizan, de manera que los análisis de microsonda nos podrán dar información sobre la petrogénesis de la roca. Una información similar se puede obtener en el caso de perfiles a través del contacto entre dos cuerpos geológicos (Figura 16).

\subsection{Limitaciones de la microsonda}

El pulido de la muestra es crítico, y los mejores resultados se obtienen en muestras muy bien pulidas. Las muestras con relieve y los bordes de granos pueden dar resultados muy bajos o muy altos. Esto es debido a que el algoritmo de corrección ZAF asume que el haz incide perpendicularmente a la superficie de la muestra y que ésta es perfectamente plana.

Es un método relativamente lento, ya que muchas veces se requieren análisis cualitativos previos para conocer cuáles son las longitudes de onda que tenemos que discriminar. Evidentemente, los mejores resultados analíticos se obtienen generalmente en muestras de las que ya conocemos el grupo mineral al que pertenecen.

Por otra parte, y como hemos comentado anteriormente, los elementos ligeros $(\mathrm{H}, \mathrm{He}, \mathrm{Li})$ no se pueden analizar con la microsonda. Otros elementos ligeros (Be, B, C, $\mathrm{O}, \mathrm{N}, \mathrm{F}$ ) son también difíciles de analizar debido a que se absorben fuertemente en la muestra. Otros elementos como el $\mathrm{Na}$ o la $\mathrm{Ag}$ son también difíciles de analizar con precisión debido a que pueden migrar durante la excitación del haz de electrones, obteniéndose un resultado más bajo. Para evitarlo, es necesario disminuir la corriente o desenfocar el haz (en fases homogéneas también se puede mover el haz durante el análisis). En algunos casos, la presencia de agua abundante en la muestra, o de elementos volátiles, puede provocar que se pierdan algunos de estos elementos, por lo que es conveniente analizarlos primero.

Otro problema es que el haz electrónico tiene una cierta penetración. Por tanto, existe la posibilidad de que una parte de haz atraviese el cristal y excite el mineral subyacente, y en este caso el resultado sería la suma de las radiaciones emitidas por el cristal superior y el inferior y el análisis puede dar un valor muy superior a 100. Esto conlleva a una meticulosa selección previa de los puntos de análisis mediante métodos ópticos.

Finalmente, existen algunas interferencias entre las líneas de algunos elementos, aspecto que debe tenerse en cuenta si dichos elementos coexisten en el mismo mineral. Por ejemplo, es bien conocida la interferencia de la línea $K \beta$ del Ti con la $K \alpha$ del V, de modo que si ambos elementos coexisten en la misma muestra el valor de concentración obtenido para el V estará sobreestimado. Existen diferentes procedimientos para minimizar el efecto de las interferencias espectrales, que incluyen la elección de otra línea analítica, en este caso por ejemplo la línea $K \beta$ del V, o bien la aplicación de correcciones basadas en mediciones secundarias. 

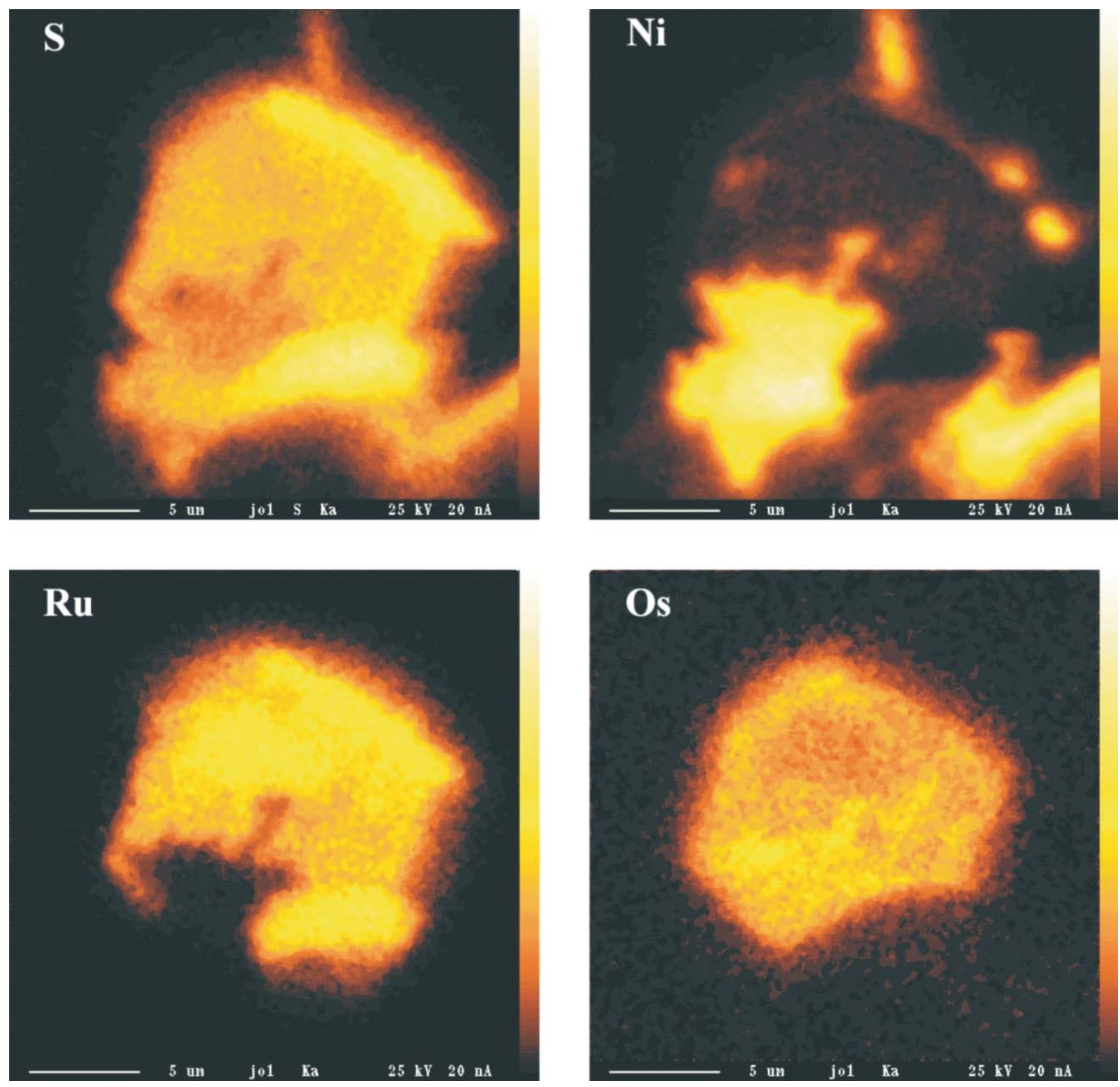

Figura 15. Mapa de intensidad de rayos X, realizados mediante microsonda electrónica, que muestra la textura y composición de un grano de laurita [(Ru,Os,Ir) $\left.\mathrm{S}_{2}\right]$ en cromititas cataclásticas del depósito Mercedita, distrito de Moa-Baracoa en Cuba Oriental (Proenza et al., 2004). Esta laurita presenta extensas variaciones composicionales a nivel de grano. Las composiciones varían desde la zona que corresponde prácticamente a laurita pura $(<1 \%$ en peso de Os y Ir; $1.05 \%$ en peso de Ni; $0.9 \%$ de $\mathrm{Rh}$ y $0.51 \%$ de As), hasta zonas donde el contenido de Ru disminuye y el de Os aumenta de forma considerable. La mayoría de los análisis realizados corresponden con laurita no estequiométrica, con muy baja proporción de $\mathrm{S}$ metal. Este déficit en azufre es producto de procesos de desulfurización de los minerales magmáticos durante la serpentinización. Los sulfuros magmáticos de Ru-Os-Ir pueden reequilibrarse durante estos procesos de alteración, para adaptarse a condiciones de menor fugacidad de oxígeno y de azufre (Garuti y Zaccarini, 1997; Proenza et al., 2007).

\section{Micro-proton-induced $x$-ray emission (Micro- PIXE)}

El micro-PIXE es una microsonda protónica. Se analizan los rayos X producidos al bombardear una muestra mediante un haz de protones que puede ser focalizado a escala micrométrica (Campbell et al., 1995). La técnica consiste básicamente en un acelerador que produce un haz de protones con una energía de $3 \mathrm{MeV}$ (aunque puede ser variable) y un sistema de focalización magnético que direcciona el haz a través de un tubo horizontal hasta la cámara donde se encuentra la muestra que va ser analizada. Unas estrictas condiciones de vacío son necesarias para evitar desenfoque del haz de protones. La muestras se 


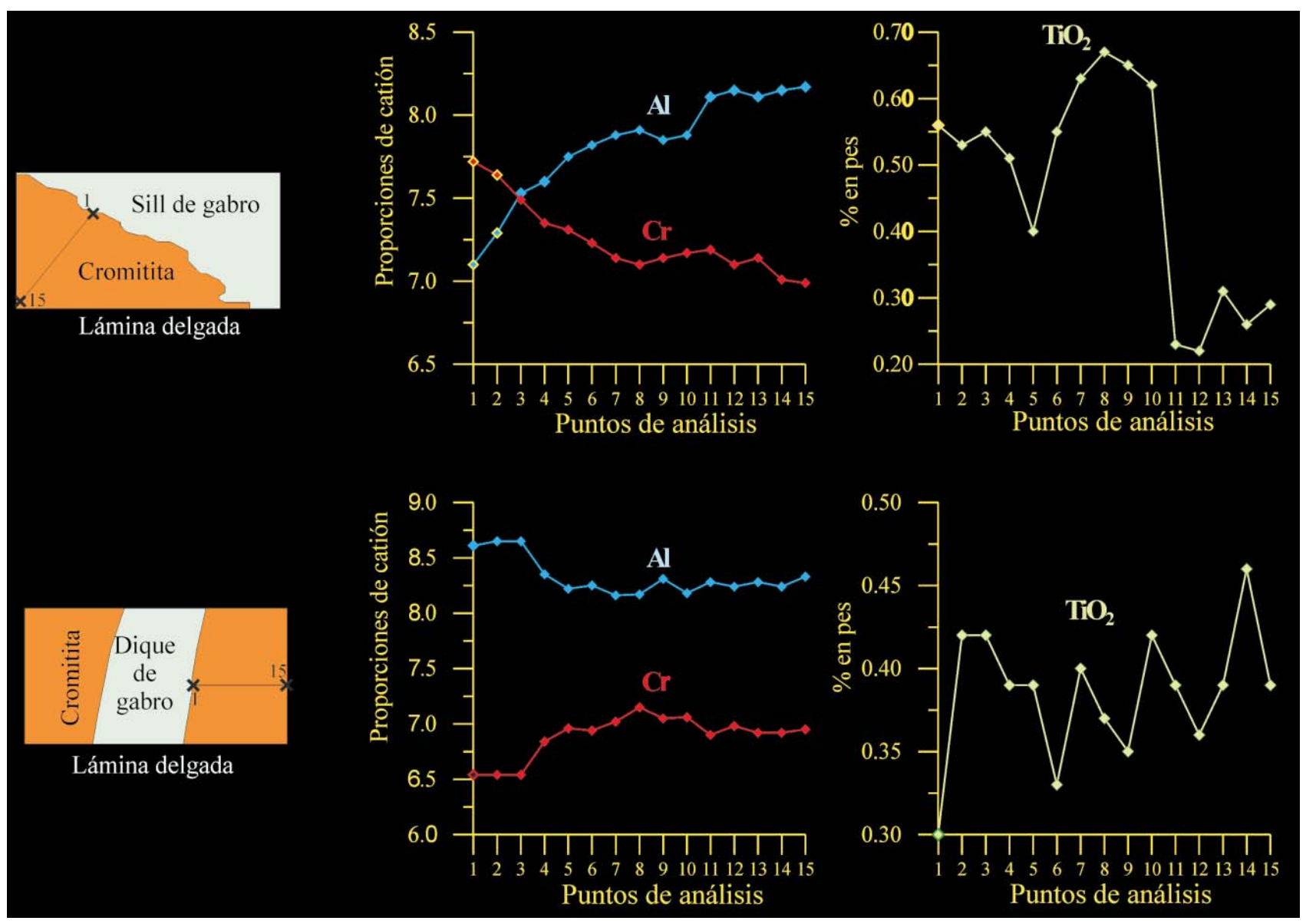

Figura 16. Perfil de microsonda realizado a escala de cuerpo geológico, en el contacto entre diques de gabro y cromititas. A la izquierda de la imagen se encuentra dibujada la lámina delgada analizada, con las unidades geológicas en estudio. Las flechas marcan la dirección de los perfiles trazados sobre la cromitita, sobre la que se analizaron 15 puntos en el segmento indicado y con el orden señalado por la flecha. A la derecha se encuentran los resultados de estos análisis. En abscisas se indica la posición de los análisis, en ordenadas la composición química expresada en proporción de catión (para el Cr y el $\mathrm{Al}$ ) o en $\%$ en peso (para el $\mathrm{TiO}_{2}$ ). Nótese que el $\mathrm{Al}$ y el $\mathrm{Cr}$ tienen un comportamiento inverso relacionado con la distancia a los cuerpos de gabro; este comportamiento es errático en el caso del titanio (Proenza et al., 1999).

preparan en forma de probetas pulidas con un diámetro de $2,5 \mathrm{~cm}$, deben tener un espesor mínimo de $50 \mu \mathrm{m}$ y estar metalizadas (generalmente con grafito). El equipo cuenta con un microscopio óptico convencional y con una cámara CCTV integrada que permite la orientación y selección de los granos que se deben analizar. El haz incide sobre la muestra con un ángulo de $45^{\circ}$, tiene un tamaño de $3 \times 6 \mu \mathrm{m}$, profundiza hasta aproximadamente $20 \mu \mathrm{m}$ y el tiempo de medida está generalmente comprendido entre 120 y 190 segundos.

Para algunos elementos, el micro-PIXE alcanza el nivel de trazas, posibilitando mapas muy detallados de distribución de elementos en un grano. La técnica tiene una gran aplicación al estudio de menas metálicas (Campbell et al., 1987; Remond et al., 1987; Cabri y Campbell, 1998). Particularmente, es muy útil para analizar cuantitativamente los elementos trazas en sulfuros (Cabri et al., 1984; Harris et al., 1984). Por ejemplo, se han obtenido muy buenos resultados en el estudio de mineralizaciones de
$\mathrm{Ni}$-Cu-(EGP), en especial la cuantificación de Pd, Rh y Ru en sulfuros, arseniuros y sulfoarseniuros (Paktunc et al., 1990; Gervilla et al., 2004). En contrapartida, debe tenerse un cuidado especial en la selección de los puntos, mayor que en el caso de la microsonda, pues el haz es más grueso y se produce una mayor penetración.

\section{Secondary-ion mass spectrometry (SIMS)}

La técnica se basa en bombardear la muestra con un haz de partículas cargadas (iones primarios); los iones arrancados de la muestra (iones secundarios) son analizados mediante un espectrómetro de masas. El método permite enfocar el haz hasta unas 10 micras de diámetro, por lo que es usado para analizar elementos traza o isótopos en minerales zonados, granos individuales de pequeño tamaño, etc. Por ejemplo, se ha comprobado en varios depósitos que el $\mathrm{Au}$ se halla en la estructura de pirita arsenical y, cuando ésta es corroída para formar arsenopirita y pirita, 
el oro queda libre en forma de micropartículas. Ejemplos de estudios de Au en sulfuros utilizando SIMS se pueden encontrar en McMahon y Cabri (1998), Cabri et al. (2000), Steele et al. (2000), Chouinard et al. (2005), Paktunc et al. (2006) y Benzaazoua et al. (2007).

\section{Laser ablation microprobe + inductively coupled plasma-mass spectrometry (LA-ICP-MS)}

Se basa en usar un láser para arrancar por ablación (sublimación) los elementos de la muestra. El haz puede ser diafragmado hasta excitar superficies muy pequeñas (del orden de pocas decenas de micras, $\sim 10 \mu \mathrm{m}$ ). El plasma así obtenido es analizado en un ICP, que disocia e ioniza el material arrancado de la muestra y transportado por una corriente de Ar, y un espectrómetro de masas (MS), que analiza las diferencias de masas de los iones producidos (Figura 17). La LA-ICP-MS es una técnica de muy alta precisión, bajos límites de detección (nivel de ppb) y sobre todo de un coste económico relativamente bajo si la comparamos con las técnicas de micro-PIXE y SIMS. El proceso de preparación de la muestra y de adquisición de los datos se realiza en un corto periodo de tiempo.

Una excelente revisión de la aplicación de la técnica de LA-ICP-MS a la resolución de problemas geológicos puede ser encontrada en Sylvester (2001). Aplicaciones concretas al estudio de depósitos minerales se pueden encontrar en Watling et al. (1995), Chenery et al., 1995, Wagner et al. (2007), Large et al. (2007), Hutchinson y McDonald (2008).

El microanálisis láser-ICP-MS tiene un enorme potencial principalmente para la determinación de elementos trazas en minerales. Desde el punto de vista de la exploración o explotación minera, esta técnica puede aplicarse fácilmente en tres grandes campos: a) análisis a microescala de elementos preciosos presentes como trazas en la estructura del mineral (oro invisible en la estructura de pirita o arsenopirita; EGP en arseniuros, etc.); b) análisis a microescala de elementos significativos en minerales indicadores (por ejemplo, análisis de $\mathrm{Ni}$ en granate de procedencia kimberlítica, para conocer la temperatura del manto subyacente, etc.), c) análisis de la distribución a microescala de elementos traza que pueden ser penalizantes desde el punto de vista metalúrgico o medioambiental.

De este modo, Wagner et al. (2007) han estudiado mediante LA-ICP-MS la distribución de Au en menas de pirita y arsenopirita procedentes del depósito Boliden (Suecia). Estas menas llegan a tener hasta $600 \mathrm{~g} / \mathrm{t}$ de Au. Las concentraciones promedio de $\mathrm{Au}$ en la arsenopirita varían entre 30 y 50 ppm y decrecen sistemáticamente con el incremento del grado de recristalización. En cambio, el contenido de Au en la pirita sistemáticamente es inferior a $0.2 \mathrm{ppm}$. Los datos de LA-ICP-MS, junto con el estudio textural, indican que el oro es liberado progresivamente de los sulfuros masivos durante el metamorfismo y reprecipitado en venas. Por otra parte, Large et al. (2007) han deter- minado, mediante LA-ICP-MS, en piritas sinsedimentarias (Sukhoi, Siberia) altos contenidos de "Au invisible" (hasta $12,2 \mathrm{ppm}$, con un valor promedio de $3.2 \mathrm{ppm}$ ) y de arsénico (1900 ppm).

Hutchinson y McDonald (2008) realizan un estudio detallado mediante LA-ICP-MS de los elementos del grupo del platino en sulfuros de la unidad Platreef (norte del complejo Bushveld). Estos autores documentan que una porción significante del $\mathrm{Pd}$, Os e Ir se encuentra en sulfuros de metales bases. El Pd está asociado principalmente a la pentlandita y la calcopirita. El Os se encuentra en solución sólida en la pirrotina y en menor medida en la pentlandita. El Ru es más abundante en la pentlandita, pero también fue detectado en la calcopirita y en la pirrotina.

La LA-ICP-MS es una técnica rápida y precisa, cada vez más común y rutinaria en diversos laboratorios de mineralogía y petrología. Como se desprende de los ejemplos de aplicaciones indicadas, su éxito se basa en su capacidad de obtención de análisis químicos casi puntuales en lámina delgada pulida o probeta. Las limitaciones que pueden apuntarse son escasas pero deben tenerse en cuenta. En primer lugar la resolución lateral del haz de láser en la mayor parte de equipos no baja de las $10 \mu \mathrm{m}$. En segundo lugar, se debe tener calibrado el equipo para los elementos deseados (lo cual no está siempre disponible de forma universal) $y$, en tercer lugar, hace falta disponer de por lo menos análisis de microsonda electrónica de uno o dos elementos presentes en el mineral.

\section{El estudio mineralógico durante las fases de exploración y explotación de depósitos minerales}

En primer lugar, desde siempre se han utilizado los concentrados minerales o de suelos durante la prospección estratégica o táctica de yacimientos minerales. El problema que se puede plantear en todos estos casos es el reconocimiento de fases con tamaño de grano muy fino, dispersas en un concentrado mineral entre otras fases mucho más abundantes. En este caso, un simple análisis visual con una lupa binocular puede no ser suficiente, perdiéndose al menos gran parte de la información. Por el contrario, en este estadio puede ser muy útil la microscopía electrónica de barrido con análisis de energías de rayos $\mathrm{X}$, especialmente cuando se dispone de un sistema de análisis de electrones retrodispersados acoplado (SEM-BSE-EDS). Este sistema puede ayudar a la localización rapidísima de granos detríticos de minerales con elementos de elevado número atómico (a efectos de interés minero directo, principalmente minerales con U, Th, Ta, Au y Pt-PGE).

En este mismo estadio, puede ser interesante determinar la proporción de un determinado mineral en el concentrado. Aunque este proceso se puede realizar de modo manual, a partir del conteo de granos bajo la lupa binocular, este mecanismo conlleva un alto grado de error, tanto mayor cuanto más complejas sean las asociaciones y cuanto más fino sea el tamaño de grano. Por otra parte, el análisis 

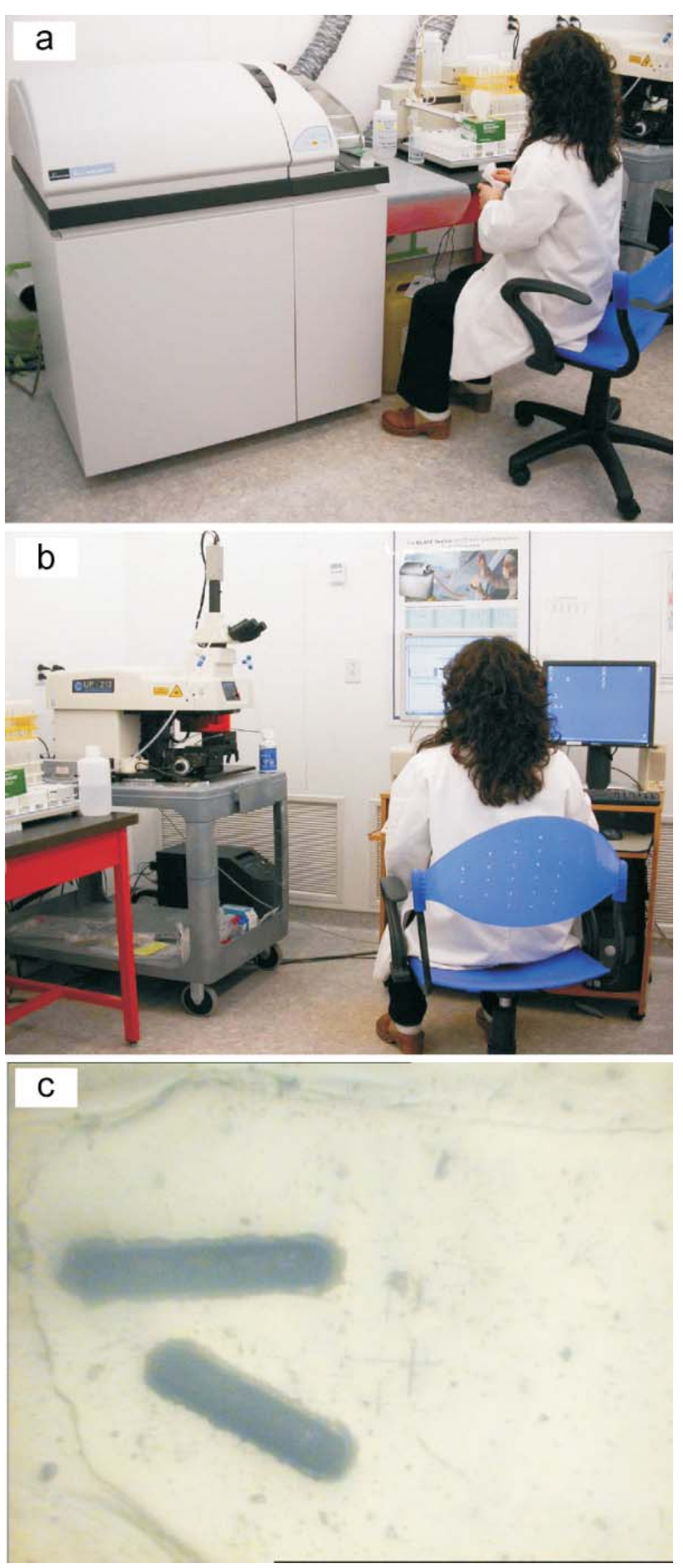

Figura 17. Equipo de LA-ICP-MS instalado en McGill University, Montreal (Québec, Canadá). A) Detalle del equipo, en el que se aprecia el espectrómetro de masas que está conectado con el microscopio. B) Detalle del microscopio óptico que lleva acoplado una cámara de video para disponer en todo momento de una imagen de la zona de análisis; el microscopio tiene acoplado un sistema de láser. C) Marcas de ablación producidas por la acción del láser sobre un cristal de granate. La forma elongada del láser se debe al desplazamiento del haz a través del cristal. Campo, $5 \mathrm{~mm}$. químico del conjunto del sedimento, suelo o concentrado dará una idea de la proporción de algunos elementos en el mismo, pero no del tipo de minerales en que cada elemento puede estar disperso. De ser preciso éste último dato, se podrían aplicar las técnicas de difracción cuantitativa de rayos X. Esta técnica puede ser una herramienta muy poderosa si es complementada eficazmente con estudios texturales con microscopía óptica de luz transmitida-reflejada, microscopía electrónica de barrido con análisis de energías y microsonda electrónica. De este modo, si conocemos la composición química de cada una de las fases minerales presentes en una mezcla, con ayuda de programas adecuados de tratamiento de los diagramas de polvo de rayos $\mathrm{X}$ pueden obtenerse con mucha precisión las proporciones en que se encuentre cada uno de estos minerales en la roca.

Por otra parte, la exploración minera de un determinado recurso se basa muchas veces en la información suministrada por ciertos minerales significativos, que se llaman “indicadores". En algunos casos, la sola presencia del mineral puede ser indicativa de la existencia de un depósito; en otros casos, es su geoquímica peculiar el dato que puede apoyar la investigación. Un ejemplo típico lo ofrece la exploración de chimeneas kimberlíticas diamantíferas. En éstas, las kimberlitas contienen, además del diamante, toda una suerte de minerales accesorios muy característicos de este tipo de asociación diamantífera, entre ellos, ilmenita magnesiana, magnesiocromita, piropo crómico, diópsido crómico. En la exploración diamantífera se concentran estos minerales y se analiza su química mineral para determinar las características geoquímicas del manto a partir del cual pueden proceder. La composición de los elementos mayores de estos minerales es obtenida rutinariamente mediante microsonda electrónica y las técnicas de caracterización de trazas son utilizadas.

Por otra parte, la determinación precisa de la composición química de la mena es fundamental para la evaluación económica y viabilidad de un proyecto minero. Por ejemplo, conocer la presencia de elementos minoritarios potencialmente recuperables que le otorguen valor añadido al producto: Au en arsenopirita, (Hinchey et al., 2003; Zacharias et al., 2004), oro en pirita (Foster et al., 2007), PGE en arseniuros (Pt-Pd en pentlandita, $\mathrm{V}$ en magnetita, Ni en goethita) o la existencia de elementos nocivos que puedan penalizar la mena en el proceso de beneficio (Mielczarski, 1999) o que generen problemas medioambientales (Subramanian et al., 2005).

\section{Conclusiones}

El estudio de las asociaciones minerales mediante técnicas cuantitativas aporta elementos valiosos para la valorización de los depósitos minerales, desde la fase de exploración a la de estudio de viabilidad del depósito.

En la fase de prospección estratégica (a nivel regional) las técnicas que pueden ser más útiles dependen del tipo de depósito. No obstante, como norma general, para 
la identificación de minerales en concentrados de batea puede ser muy útil la microscopía electrónica de barrido, especialmente en el modo de electrones retrodispersados y acompañada de microanálisis por dispersión de energías. Este método permitirá la localización e identificación rápida de partículas de minerales pesados en el concentrado. En este estadio la microsonda electrónica u otras técnicas de microanálisis cuantitativo (LA-ICP-MS, SIMS, MicroPIXE u otras) sólo pueden ser viables en el caso de que el tipo de depósito que se trata de localizar presente un fuerte valor añadido, como es en la exploración de depósitos de diamante a partir de la composición de minerales indicadores.

Por lo común, el estudio del depósito en la fase táctica (ya a nivel local o del depósito) precisará de herramientas aplicadas a un espacio mucho más concreto y, por tanto, que deben ser precisas. En este estadio la difracción de polvo de rayos $\mathrm{X}$ cuantitativa puede ser una alternativa $\mathrm{o}$ un complemento a otros métodos (PIMA, p. ej.) enfocados a caracterizar la distribución de las zonas de alteración hidrotermal o meteórica, o a caracterizar la mineralogía de suelos. Nuevamente la microscopía electrónica de barrido puede ser un complemento eficaz para identificar los minerales de interés económico.

En la fase de valoración del depósito pueden emplearse las dos técnicas citadas, pero en este estadio puede ser ya imperativo trabajar con técnicas cuantitativas más detalladas, incluyendo las microsondas electrónicas, iónicas o protónicas, así como la LA-ICP-MS. Debido a su elevado coste, es posible que las tres últimas técnicas sólo sean rentables en el caso de que el mineral explotable tenga un alto valor añadido. En el caso de la exploración de diamante, no obstante, estas técnicas son esenciales para poder definir el potencial diamantífero de una kimberlita.

Las técnicas mineralógicas reseñadas pueden ser, finalmente, aplicadas al diseño de la explotación del depósito, e incluso al control de seguimiento del mismo. En particular, el estudio de la textura de las menas (especialmente, el tamaño de grano y los tipos de intercrecimientos) es esencial en el diseño de la trituración de las mismas. Este aspecto puede ser estudiado con mucho provecho en microscopía electrónica de barrido con análisis de energías.

A efectos también de mecanismos de beneficio, así como de control del posible impacto medioambiental, el tipo de mena y su distribución han de ser caracterizados de manera segura y detallada, aspecto que puede ser realizado con ayuda del conjunto de las técnicas citadas. Así, la difracción de polvo cuantitativa puede ser una herramienta poderosa para controlar la distribución del mineral en el depósito. Ello implica, indirectamente, conocer la distribución de los elementos químicos mayores. Esta técnica no aporta el mismo tipo de datos que el que ofrece un típico análisis geoquímico como los comúnmente usados por las empresas para la determinación de la ley de un elemento en el depósito (fluorescencia de rayos X, activación neutrónica, ICP-MS, etc.), pero presenta la ventaja de ofrecer en su lugar la ley de mineral en el mismo depósito. Ello tiene algunas ventajas importantes, pues un cierto elemento puede estar distribuido entre diversos minerales y si en alguno de los minerales no fuera económica la extracción del elemento el análisis químico clásico falsearía el planteamiento de las técnicas mineralotécnicas y metalúrgicas que deben ser empleadas, e incluso la viabilidad de la explotación. El mismo razonamiento puede ser aplicado para demostrar la validez de aplicar el SEM-EDS al diseño de la planta de beneficio de un depósito. En particular, los elementos preciosos y otros con alto valor añadido $(\mathrm{U}$, $\mathrm{Nb}, \mathrm{Ta} . .$.$) pueden ser explotables a leyes muy bajas, pero$ siempre que las menas tengan unas características mineralógicas favorables. En particular, debe conocerse con precisión cuál es la distribución del elemento o elementos beneficiables entre las diferentes especies minerales del depósito. Para ello pueden ser imprescindibles análisis con microsonda electrónica e incluso micro-PIXE, SIMS o LA-ICP-MS.

Para el diseño de los mecanismos de control medioambiental deben tenerse también en cuenta las características químicas y físicas de las menas, en particular, aquéllas que puedan favorecer su alterabilidad. Además, es importante conocer la distribución de algunos elementos traza tóxicos $(\mathrm{Cd}, \mathrm{Cr}, \mathrm{Bi}, \mathrm{Se}, \mathrm{Tl}$, etc.) entre los diferentes minerales del depósito, no en el conjunto del depósito (con los métodos químicos tradicionales). Nuevamente, también en este caso las técnicas de microanálisis puntual mineralógico (EMP, micro-PIXE, SIMS, LA-ICP-MS) pueden ser extremadamente seguras y eficaces.

\section{Agradecimientos}

Este trabajo es una contribución a un proyecto internacional de cooperación al desarrollo de la Universidad de Barcelona (Vicerectorat de Política i Mobilitat). Este trabajo ha sido mejorado gracias a los comentarios y sugerencias de F. Zaccarini, G. Garuti y M. Escayola.

\section{Referencias}

Bish, D.L., Post, J.E. (eds.) 1989, Modern Powder Diffraction, MSA Reviews in Mineralogy 20: Washington, Mineralogical Association of America, $384 \mathrm{p}$.

Bowles, J.F.W., 2002, Application of electron microbeam analysis in the earth sciences: Mikrochimica Acta, 138, 125-131.

Benzaazoua, M., Marion, P., Robaut, F., Pinto, A., 2007, Gold-bearing arsenopyrite and pyrite in refractory ores: analytical refinements and new understanding of gold mineralogy: Mineralogical Magazine, 71,123-142.

Cabri, L.J., Blank, H., El Goresy, A., Laflamme, J.H.G., Nobiling, R., Sizgoric, M.B., Traxel, K., 1984, Quantitative trace-element analyses of sulfides from Sudbury and Stillwater by proton microprobe: Canadian Mineralogist, 22, 521-542.

Cabri, L.J., Campbell, J.L., 1998, The proton microprobe in ore mineralogy (micro-PIXE) technique), in Cabri, L.J., Vaughan, D.J. (eds.) Modern Approaches to Ore and Environmental Mineralogy: Ottawa, Canada, Mineralogy Association of Canada, 181-198.

Cabri, L.J., Newville, M., Gordon, R.A., Crozier, E.D., Sutton, S.R., 
Mahon, G., Jiang, D-T., 2000, Chemical speciation of gold in arsenopyrite: Canadian Mineralogist, 38, 1265-1281.

Campbell, J.L., Cabri, L.J., Rogers, P.S.Z., Traxel, K., Benjamin, T.M., 1987, Calibration of micro-PIXE analysis of sulfide minerals Nuclear Instruments and Methods in Physics Research Section B, B22, 437-441.

Campbell, J.L., Teesdale, W.J., Halden, N.M., 1995, Theory, practice and application of PIXE micro-analysis and SPM element mapping Canadian Mineralogist, 33, 279-292.

Castaing, R., Guinier, A., 1950, Application des sondes electroniques a l'analyse metallographique: First International Conference on Electron Microscopy, 1949: Delft, 60-63.

Chenery, S., Cook, J.M., Styles, M., Cameron, E.M., 1995, Determination of the three-dimensional distributions of precious metals in sulphide minerals by laser ablation microprobe-inductively coupled plasma-mass spectrometry (LAMP-ICP-MS ): Chemical Geology, 124, 55-65.

Chouinard, A., Paquette, J., Williams-Jones, A.E., 2005, Crystallographic controls on trace-element incorporation in auriferous pyrite from the Pascua epithermal high-sulfidation deposit, Chile-Argentina: Canadian Mineralogist, 43, 951-963.

Cocherie, A., Legendre, O., Peucat, J.J., Kouamelan, A.N., 1998 , Geochronology of polygenetic monazites constrained by in situ electron microprobe Th-U-total lead determination; implications for lead behaviour in monazite: Geochimica Cosmochimica Acta., 62, 2475-2497.

Coy-YII, R., 1970, Quelques aspects de la cahodoluminescence des minéraux: Chemical Geology, 5, 243-254

Cullity, B.D., Stock, S.R., 2001, Elements of X - Ray Diffraction: Reading, Massachusetts, Addison-Wesley, $664 \mathrm{p}$.

Díaz-Martínez, R., Proenza, J.A., Comas, J., Fernández-Bellon, O., Fabra, J.M., Guinart, O., Melgarejo, J.C., 1998, El placer lateral de playa Mejías (noreste de Cuba Oriental): Un ejemplo de interacción de procesos aluviales y marinos en la concentración de minerales de elementos preciosos: Acta Geologica Hispanica, 33, 351-371.

Ebers, M.L., Kopp, O.C., 1979, Cathodolumineseent microstratigraphiyn gangue dolomite, the Mascot-Jefferson City district, Tennessee: Economic Geology, 74, 908-918.

Esteve, V. (ed.) 2006, El método Rietveld: Publicacions de la Universita Jaume I, Colección "Ciències experimentals",9, 192 p.

Foster, A.R., Williams, P.J., Ryan, C.G., 2007, Distribution of gold in hypogene ore at the Ernest Henry iron oxide copper-gold deposit, Cloncurry district, NW Queensland: Exploration \& Mining Geology, 16, 125-143.

Garuti, G., Zaccarini, F., 1997, In situ alteration of platinum-group minerals at low temperature; evidence from serpentinized and weathered chromitite of the Vourinos Complex, Greece: Canadian Mineralogist, 35, 611-626.

Gervilla, F., Cabri, L.J., Kojonen, K., Oberthur, T., Weiser, T.W., Johanson, B., Sie, S.H., Campbell, J.L., Teesdale, W.J., Gilles Laflamme, J.H., 2004, Platinum-Group Element Distribution in Some Ore Deposits: Results of EPMA and Micro-PIXE Analyses: Microchimica Acta, 147, 167-173.

Harris, D.C., Cabri, L.J., Nobiling, R., 1984, Silver-bearing chalcopyrite, a principal source of silver in the Izok Lake massive sulphide deposit. Confirmation by electron- and probe-microprobe:Contributions to Mineralogy and Petrology, 110, 1-15.

Hinchey, J.G., Wilton, D.H.C., Tubrett, M.N.A., 2003, A LAM-ICP- MS study of the distribution of gold in arsenopyrite from the Lodestar prospect, Newfoundland, Canada: Canadian Mineralogist, 41, 353-364.

Hutchinson, D., McDonald, I., 2008, Laser ablation ICP-MS study of platinum-group elements in sulphides from Platreef at Turfspruit, northern limb of the Bushveld Complex, South Africa: Mineralium Deposita, 43, 695-711.

Klug, H.P., Alexander, L.E., 1954, X-Ray Diffraction Procedures for polycrystalline and amorphous materials: New York, John Wiley and Sons, $716 \mathrm{p}$.
Large, R.R., Maslennikov, V.V., Robert, F., Danyushevsky, L.V., Chang, Z., 2007, Multistage Sedimentary and Metamorphic Origin of Pyrite and Gold in the Giant Sukhoi Log Deposit, Lena Gold Province, Russia: Economic Geology, 102, 1233-1267.

Marshall, D.J., 1988, Cathodoluminescence of geologic materials: Boston, Unwin Hyman, $146 \mathrm{p}$.

McMahon, G., Cabri, L.J., 1998: The SIMS in ore mineralogy, in Cabri, L.J., Vaughan, D.J. (eds.) Modern Approaches to Ore and Environmental Mineralogy: Ottawa, Canada, Mineralogy Association of Canada, 199-224.

McGee, J.J., Keil, K., 2001, Application of electron probe microanalysis to the study of geological and planetary materials: Microscopy and Microanalysis, 7, 200-210.

Mielczarski, J.A., Cases, J., Mielczarski, E., Marion, P., Fordham, M., Valente, C., 1999, Production of clean copper sulfide concentrate, in Gaballah, I., Hagel, J., Solozabal, R. (eds.) REWAS '99-Global Symposium on Recycling, Waste Treatment and Clean Technology: Pennsylvania, U.S.A., TMS, 1929-1939.

Paktunc, A.D., Hulbert, L.J., Harris, D.C., 1990, Partitioning of platinumgroup and other trace elements in sulfides from the Bushveld Complex and Canadian occurrences of nickel-copper sulfides: Canadian Mineralogist, 28, 475-488.

Paktunc, A.D., Kingston, D., Pratt, A., McMullen, J., 2006, Distribution of gold in pyrite and in products of its transformation resulting from roasting of refractory gold ore: Canadian Mineralogist, 44, 213-227.

Proenza, J., Gervilla, F., Melgarejo, J.C., Bodinier, J.L., 1999, Al- and Cr-rich chromitites from the Mayarí-Baracoa ophiolitic belt (eastern Cuba); consequence of interaction between volatile-rich melts and peridotites in suprasubduction mantle: Economic Geology, 94, 547-566.

Proenza, J.A., Rodríguez-Vega, A., Díaz-Martínez, R., Gervilla, F., Melgarejo, J.C., Ramayo, L., Vila, A.R., 2004, Distribución de elementos del grupo del platino (EGP) y Au en la faja ofiolítica Mayarí-Baracoa (Cuba oriental), en Pereira, E.S., Castroviejo, R., Ortiz, F. (eds.), Complejos ofiolíticos en Iberoamérica: guías de prospección para metales preciosos: Madrid, Proyecto XIII.1Ciencia y Tecnología Para el Desarrollo, 309-336.

Proenza, J.A., Zaccarini, F., Lewis, J., Longo, F., Garuti, G., 2007, Chromite composition and platinum-group mineral assemblage of PGE-rich Loma Peguera chromitites, Loma Caribe peridotite, Dominican Republic: Canadian Mineralogist, 45, 631-648.

Reed, S.J.B., 2005, Electron microprobe analysis and scanning electron microscopy in geology: Cambridge, U.K., Cambridge University Press, 201p.

Remond, G., Cesbron, F., Traxel, K., Campbell, J.L., Cabri, L.J., 1987, Electron microprobe analysis and protron induced X-ray spectrometry applied to trace elements in sulfides: problems and prospects: Scanning Microscopy, 1, 1017-1037.

Robinson, B.W., Ware, N.G., Smith, D.G.W., 1998, Modern electronmicroprobe trace-elements analysis in mineralogy, in Cabri, L.J., Vaughan, D.J. (eds.) Modern Approaches to Ore and Environmental Mineralogy: Ottawa, Canada, Mineralogy Association of Canada, 153-180.

Steele, I.M., Cabri, L.J., Gaspar, J.C., McMahon, G., Marquez, M.A., Vasconcellos, M.A.Z., 2000, Comparative analysis of sulfides for gold using SXRF and SIMS: Canadian Mineralogist, 38, 1-10.

Subramanian, K.N., Connelly, D.E.G., Wong, K.Y., 2005, Separation of pyrite and arsenopyrite in a gold sulfide concentrate, in Centenary of Flotation Symposium: Brisbane, Australia, Australasian Institute of Mining and Metallurgy, 1045-1052.

Sylvester, P., 2001, Laser-ablation-ICPMS in the earth sciences: Ottawa, Mineralogical Association of Canada Short Course Series Vol. 29, $243 \mathrm{p}$.

Wagner, T., Klemd, R., Wenzel, T., Mattsson, B., 2007, Gold upgrading in metamorphosed massive sulfide ore deposits: Direct evidence from laser-ablation-inductively coupled plasma-mass spectrometry analysis of invisible gold: Geology, 35, 775-778. 
Watling, R.J., Herbert, H.K., Barrow, I.S., Thomas, A.G., 1995, Analysis of diamonds and indicator minerals for diamond exploration by laser ablation-inductively coupled plasma mass spectrometry: Analyst, 120, 1357-1364.

Williams, M.L., Jercinovic, M.J., Terry, M.P., 1999, Age mapping and dating of monazite on the electron microprobe: deconvoluting multistage tectonic histories: Geology, 27, 1023-1026.
Zacharias, J., Fryda, J., Paterova, B., Mihaljevic, M., 2004, Arsenopyrite and As-bearing pyrite from the Roudny deposit, Bohemian Massif: Mineralogical Magazine, 68, 31-46.

Recibido: 22/10/2008

Recibido corregido: 30/04/2009

Aceptado: 04/05/2009 